\title{
Do Subjective Expectations Explain Asset Pricing Puzzles?*
}

\author{
Gurdip Bakshi $^{\mathrm{a} \dagger}$ Georgios Skoulakis $\mathrm{b} \neq$

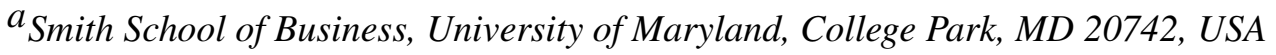

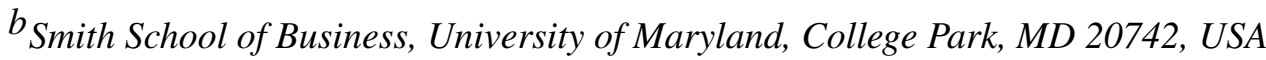

First draft, May 2008

This draft, January 7, 2009

\begin{abstract}
The structural uncertainty model with Bayesian learning, advanced by Weitzman (AER 2007), provides a framework for gauging the effect of structural uncertainty on asset prices and risk premiums. This paper provides an operational version of this approach that incorporates realistic priors about consumption growth volatility, while guaranteeing finite asset pricing quantities. In contrast to the extant literature, the resulting asset pricing model with subjective expectations yields well-defined expected utility, finite moment generating function of consumption growth, and tractable expressions for equity premium and riskfree return. Our quantitative analysis reveals that explaining the historical equity premium and riskfree return, in the context of subjective expectations, requires implausible levels of structural uncertainty. Furthermore, these implausible prior beliefs result in consumption disaster probabilities that virtually coincide with those implied by more realistic priors. At the same time, the two sets of prior beliefs have diametrically opposite asset pricing implications: one asserting, and the other contradicting, the antipuzzle view.
\end{abstract}

KEY WORDS: subjective expectations; learning; structural uncertainty; priors; predictive density of consumption growth; equity premium; riskfree return.

JEL Classification Codes: D34, G12.

\footnotetext{
${ }^{*}$ The authors acknowledge helpful discussions with Isabelle Bajeux-Besnainou, Alexandre Baptista, Dobrislav Dobrev, Lorenzo Garlappi, Steve Heston, Jerry Hoberg, Nengjiu Ju, Pete Kyle, Dilip Madan, Anna Obizhaeva, Nagapurand Prabhala, Matt Pritsker, Mark Loewenstein, Jay Shanken, Steve Sharpe, and especially Martin Weitzman. Earlier versions of the paper were presented at the Federal Reserve Board, University of Maryland, George Washington University, and 2008 Latin American Meeting of the Econometric Society (LAMES), Rio de Janeiro, Brazil. We welcome comments, including references to related papers we have inadvertently overlooked.

${ }^{\dagger}$ Tel.: +1-301-405-2261; fax: +1-301-405-0359. E-mail address: gbakshi@ rhsmith.umd.edu

†Tel.: +1-301-405-2934; fax: +1-301-405-0359. E-mail address: gskoulak@rhsmith.umd.edu
} 
The structural uncertainty model with Bayesian learning, developed by Weitzman (2007), provides an economic environment for understanding the effect of structural uncertainty on asset prices and risk premiums. Rooted in the theoretical underpinnings of Lucas (1978), the logical structure of the model formalizes the sense in which structural parameter uncertainty can dominate aggregate consumption risk in explaining aggregate stock market behavior. ${ }^{1}$

In one starkly revealing characterization, Weitzman (2007) shows that when the representative agent experiences uncertainty about the volatility of consumption growth, the support of which is assumed to be the entire positive real line, and updates his beliefs as a Bayesian, then the predictive consumption growth distribution, which is Normal in the absence of structural uncertainty, gets transformed into a predictive heavy-tailed Student-t distribution. This theoretical modification within the Lucas paradigm is enormous in its asset pricing implications. First, the theoretical results imply an infinite equity risk premium suggesting that the agent demands large compensation for bearing uncertainty about the consumption growth volatility, the true structure of which remains unknown forever. Second, the observed magnitudes of equity risk premium and riskfree return are not to be regarded as puzzles requiring reconciliation, but rather as antipuzzles. The effect of structural uncertainty in a Bayesian framework is sufficiently powerful to reverse the direction of asset pricing puzzles, implying the futility of aligning theoretical models to fit observed asset pricing quantities.

How general is the property of being able to produce a large equity premium by incorporating structural uncertainty and learning in the model of Lucas (1978)? Is it a feature that is intrinsic to the predictive Student-t distribution for consumption growth, for which the moment generating function does not exist? Are theoretical implications robust when one substitutes the Student-t distribution with a comparable class of heavy-tailed Bayesian predictive distributions? At the heart of our contribution is an exact characterization of the predictive distribution of consumption growth that supports the finiteness of the moment

\footnotetext{
${ }^{1}$ There is a long list of theoretical and empirical research based on the consumption based asset pricing model. See for instance, Lucas (1978), LeRoy and Porter (1981), Mehra and Prescott (1985), Campbell (1986), Rietz (1988), Epstein and Zin (1991), and Campbell and Cochrane (1999, 2000). Related treatments and refinements are exposited in Weil (1989), Campbell (1993), Cecchetti, Lam, and Mark (2000), Abel (2002), Aït-Sahalia, Parker, and Yogo (2004), Bansal and Yaron (2004), Barro (2006), Gabaix (2007), Lettau and Wachter (2007), Lettau, Ludvigson, and Wachter (2007), and Cogley and Sargent (2008). Overview of these approaches are provided in Mehra and Prescott (2003, 2008), and Kocherlakota (1996). A strand of contributions in Bayesian asset pricing model building and learning include Timmermann (1993, 1996), Brennan and Xia (2001), Lewellen and Shanken (2002), Tsionas (2005), Jobert, Platania, and Rogers (2006), Cogley (2007), and Adam, Marcet, and Nicolini (2008).
} 
generating function. The operational version of the theory we offer is imperative to ensuring finite expected utility (e.g., Geweke (2001)) and for studying the quantitative asset pricing implications of the model, when the support of consumption growth volatility is a bounded interval.

We operationalize the theory by accommodating prior and posterior distributions of the precision of consumption growth that are both in the truncated Gamma class. The linchpin of our approach is a theorem that establishes the conjugacy of the prior and the posterior distributions for the precision of consumption growth, under a judicious choice of a transition mechanism. The derived predictive density of consumption growth is akin to the Student-t distribution in terms of their probabilistic laws, except possessing finite moments of all orders. The theoretical model offers the advantage of a representation of asset pricing quantities under structural uncertainty and Bayesian learning that is amenable to convenient computation through the moment generating function. Our approach can be construed as a mathematical formalization of an asset pricing model that incorporates a compact support for consumption growth volatility, and addresses the intuition conveyed in Weitzman (2007) on the role of subjective expectations in determining asset prices.

The model with structural uncertainty is implemented to investigate its potential to resolve asset pricing puzzles. Our quantitative exercises yield three main conclusions. First, we observe that plausible structural uncertainty, summarized by the uncertainty about consumption growth volatility, fails to produce a large equity premium. Corresponding to maximum allowable level of consumption growth volatility of 50\% and a risk aversion of 3 , the equity premium generated by the model is $0.13 \%$. Second, the response of asset pricing quantities is flat over a broad range of configurations of structural uncertainty. Only when the maximum level of consumption growth volatility is unreasonably high can the model match the average equity premium and the average riskfree return of $6 \%$ and $1 \%$, respectively. Third, we find that two sets of priors that result in consumption disaster probabilities that are indistinguishable nevertheless generate vastly different asset pricing implications. The gist is that rationalizing asset pricing phenomena through subjective expectations demands arguably excessive levels of structural uncertainty.

The paper proceeds as follows. Section I outlines the Weitzman (2007) framework for understanding the asset pricing implications of structural uncertainty. Section II departs from Weitzman (2007) and pro- 
vides an alternative characterization of the predictive density of consumption growth that supports a finite moment generating function. The generalization posits uncertainty about consumption growth volatility over a plausible compact support instead of the entire positive real line. The focus of Section III is to investigate whether structural uncertainty with realistic priors facilitates a resolution, or exacerbates, welldocumented asset pricing puzzles. Finally, Section IV concludes the paper. Proofs are in the Appendix.

\section{Structural Uncertainty Framework for Understanding Asset Pricing Puz- zles in Weitzman (2007)}

The following assumptions about preferences, subjective expectations, and learning about structural uncertainty are at the center of the theoretical analysis in Weitzman (2007).

Assumption 1 The model is developed in terms of a representative agent who orders his preferences over random consumption paths, and maximizes utility subject to the usual budget constraint (Lucas (1978)),

$$
E_{t}\left(\sum_{j=0}^{\infty} \beta^{j} U\left(C_{t+j}\right)\right), \quad \text { where } \quad U\left(C_{t}\right)=\frac{C_{t}^{1-\alpha}}{1-\alpha}
$$

$C_{t}$ denotes consumption at time $t, 0<\beta<1$ is the time-preference rate, and $E_{t}($.$) is expectation operator$ with respect to the subjective distribution of future consumption growth rates. The agent has power utility function with coefficient of relative risk aversion $\alpha \in \mathfrak{R}^{+}$.

The assumption about time-separable power utility is standard and follows from the earlier contributions of Merton (1969), Lucas (1978), Mehra and Prescott (1985), Cox, Ingersoll, and Ross (1985), and Campbell (1986). The present model differs from the previous literature in that it accommodates different

probability beliefs as reflected in subjective expectations about consumption growth. There is uncertainty in the economy with respect to the stochastic process of consumption growth,

$$
X_{t+1} \equiv \ln \left(\frac{C_{t+1}}{C_{t}}\right), \quad X_{t+1} \in \Re
$$


In particular, the volatility of consumption growth, denoted by $\sigma_{t}$, is stochastic and unobservable. Define the precision as

$$
\theta_{t} \equiv \frac{1}{\sigma_{t}^{2}} \in(0, \infty)
$$

which is the reciprocal of the variance of consumption growth.

Suppose observations on realized consumption growth are available starting in period $\tau$, which should be thought of as representing the distant past. For each time period $t=\tau, \tau+1, \ldots$, denote by $X^{t}$ the set of all observations on consumption growth up to and including period $t: X^{t}=\left\{x_{\tau}, \ldots, x_{t}\right\}$.

The intertemporal marginal rate of substitution between $t$ and $t+1$ is given by $\beta\left(\frac{C_{t+1}}{C_{t}}\right)^{-\alpha}=\beta e^{-\alpha X_{t+1}}$. At each time $t$, the agent obtains the subjective distribution of the consumption growth rate $X_{t+1}$ by conditioning on all past data $X^{t}$.

Assumption 2 Conditional on precision $\theta_{t}$, the consumption growth, $X_{t}$, is Normally distributed,

$$
X_{t} \sim N\left(\mu, \frac{1}{\theta_{t}}\right), \quad \text { with } \quad p\left(X_{t} \mid \theta_{t}\right)=\frac{1}{\sqrt{2 \pi}} \theta_{t}^{\frac{1}{2}} e^{-\frac{\theta_{t}\left(X_{t}-\mu\right)^{2}}{2}},
$$

where $p\left(X_{t} \mid \theta_{t}\right)$ denotes the density of consumption growth conditional on $\theta_{t}$. The mean consumption growth is $\mu$, which is assumed to be a known constant.

It must be appreciated that the Weitzman (2007) framework can be refined to allow for learning about mean consumption growth without affecting model predictions about asset pricing puzzles. In particular, Theorem 2.25 in Bauwens, Lubrano, and Richard (1999) shows that when both the mean and the variance are unknown, the resulting predictive distribution is Student-t, but with inflated variance. Furthermore, Example 3 in Geweke (2001) emphasizes that in a model with known variance and unknown mean, the predictive and the primitive consumption growth distributions are both Gaussian. It is learning about volatility, as opposed to learning about the mean, that is fundamental to generating fat-tailed predictive consumption growth distributions important for addressing the asset pricing issues at hand.

The agent is uncertain about the true $\theta_{t+1}$ and maintains subjective beliefs captured by the conditional density $p\left(\theta_{t+1} \mid X^{t}\right)$ to be described shortly. The modeling innovation of Weitzman (2007) is that the agent 
makes Bayesian inferences about $\theta_{t+1}$ given history $X^{t}$, combining elements from Harvey and Fernandes (1989), Shephard (1994), and Geweke (2001).

Assumption 3 Assume that, in the initial period $\tau$, the conditional density, $p\left(\theta_{\tau} \mid X^{\tau}\right)$, is Gamma $\left(a_{\tau}, b_{\tau}\right)$. Specifically,

$$
p\left(\theta_{\tau} \mid X^{\tau}\right)=\frac{b_{\tau}^{a_{\tau}}}{\Gamma\left[a_{\tau}\right]} \theta_{\tau}^{a_{\tau}-1} e^{-b_{\tau} \theta_{\tau}}, \quad \theta_{\tau} \in(0, \infty), \quad a_{\tau}>0, \quad b_{\tau}>0
$$

where $\Gamma[n]=\int_{0}^{\infty} z^{n-1} e^{-z} d z$ is the complete Gamma function with $n>0$.

The evolution of precision $\theta_{t}$ is described in the following sequential fashion so as to maintain the conjugacy of the system through time.

Assumption 4 Given precision of consumption growth $\theta_{t}$, the evolution of precision at date $t+1, \theta_{t+1}$, is governed through the transition equation,

$$
\theta_{t+1}=\frac{1}{\omega} \eta_{t+1} \theta_{t}, \quad 0<\omega<1,
$$

where the multiplicative shock $\eta_{t+1}$, given the history $X^{t}$, follows a $\operatorname{Beta}\left(\omega a_{t},(1-\omega) a_{t}\right)$ distribution.

Equation (6) specifies the learning scheme about structural uncertainty in the economy and is the driving force behind the fatter tails of the predictive distribution of consumption growth. The parameter $\omega$ is a constant that controls the speed of precision. An important aspect of the above specification, as adopted in Weitzman (2007), is that the support of the precision process is the entire positive real line. As a result, the consumption growth volatility can take arbitrarily large values with positive probability.

Under Assumptions 3 and 4, it follows from the analysis in Section 3 in Shephard (1994) that the conditional density, $p\left(\theta_{t+1} \mid X^{t}\right)$, remains in the Gamma family and preserves conjugacy. Specifically, it follows that (the notation of $a_{t \mid t-1}$ and $b_{t \mid t-1}$ in Shephard (1994) is replaced by $A_{t-1}$ and $B_{t-1}$, respectively):

$$
\theta_{t} \mid X^{t} \sim \operatorname{Gamma}\left(a_{t}, b_{t}\right) \quad \text { and } \quad \theta_{t} \mid X^{t-1} \sim \operatorname{Gamma}\left(A_{t-1}, B_{t-1}\right),
$$


where $a_{t}, b_{t}$ and $A_{t-1}, B_{t-1}$ are related via the recursive equations,

$$
a_{t}=A_{t-1}+\frac{1}{2}, \quad A_{t-1}=\omega a_{t-1}, \quad b_{t}=B_{t-1}+\frac{1}{2}\left(x_{t}-\mu\right)^{2}, \quad B_{t-1}=\omega b_{t-1} .
$$

Note that the recursions involving $A_{t}$ and $B_{t}$ alone are expressed as

$$
A_{t}=\omega\left(A_{t-1}+\frac{1}{2}\right), \quad B_{t}=\omega\left(B_{t-1}+\frac{1}{2}\left(x_{t}-\mu\right)^{2}\right) .
$$

Moreover, recursive substitution in terms of past history $\tau$ of consumption growth yields:

$$
\begin{aligned}
& A_{t}=\frac{1}{2}\left(\omega+\cdots+\omega^{t-\tau}\right)+\omega^{t-\tau} A_{\tau}=\frac{\omega}{2}\left(\frac{1-\omega^{t-\tau}}{1-\omega}\right)+\omega^{t-\tau} A_{\tau}, \\
& B_{t}=\frac{1}{2}\left(\omega\left(x_{t}-\mu\right)^{2}+\omega^{2}\left(x_{t-1}-\mu\right)^{2}+\cdots+\omega^{t-\tau}\left(x_{\tau+1}-\mu\right)^{2}\right)+\omega^{t-\tau} B_{\tau} .
\end{aligned}
$$

In the spirit of Lemma 5 of Weitzman (2007), assume the availability of a large history on consumption growth starting in period $\tau$ and let $\omega=(k-1) / k$, where the parameter $k$ captures the effective sample size. Imposing a large (in absolute value) $\tau<0$ in (10)-(11) and noting that $0<\omega<1$, the following relationships are obtained:

$$
A_{t} \approx \frac{\omega}{2(1-\omega)}=\frac{k-1}{2}, \quad B_{t} \approx \frac{(k-1) V_{t}}{2}, \quad \text { where } V_{t}=\sum_{j=0}^{t-\tau-1} \frac{1}{k}\left(1-\frac{1}{k}\right)^{j}\left(x_{t-j}-\mu\right)^{2}
$$

Thus, $2 A_{t} \approx k-1$ and $B_{t} A_{t}^{-1} \approx V_{t}$. Hinging on a large history of past realizations of consumption growth, $V_{t}$ is a state variable that represents the volatility of consumption growth with declining weights $\frac{1}{k}\left(1-\frac{1}{k}\right)^{j}$.

With the aforementioned structural uncertainty and the posited Bayesian learning rule, the theoretical object of interest is the predictive distribution of $X_{t+1} \in \mathfrak{R}$ given all past observations:

$$
\begin{aligned}
g\left(X_{t+1} \mid X^{t}\right) & =\int p\left(X_{t+1} \mid \theta_{t+1}, X^{t}\right) p\left(\theta_{t+1} \mid X^{t}\right) d \theta_{t+1}=\int p\left(X_{t+1} \mid \theta_{t+1}\right) p\left(\theta_{t+1} \mid X^{t}\right) d \theta_{t+1} \\
& \propto\left(1+\frac{\left(X_{t+1}-\mu\right)^{2} /\left(B_{t} A_{t}^{-1}\right)}{2 A_{t}}\right)^{-\left(\frac{2 A_{t}+1}{2}\right)} \quad \text { (Weitzman (2007), Eq. 24). }
\end{aligned}
$$


Therefore, the Bayesian predictive distribution can be characterized as the fat-tailed Student-t distribution, with degrees of freedom $2 A_{t}$, represented by,

$$
Y_{t+1} \sim \mathrm{t}\left(2 A_{t}\right), \quad \text { where } Y_{t+1} \equiv \frac{X_{t+1}-\mu}{\sqrt{B_{t} A_{t}^{-1}}} \in \Re
$$

with density

$$
g\left(Y_{t+1} ; 2 A_{t}\right)=\frac{\Gamma\left[\left(2 A_{t}+1\right) / 2\right]}{\sqrt{\pi 2 A_{t}} \Gamma\left[A_{t}\right]}\left(1+\frac{Y_{t+1}^{2}}{2 A_{t}}\right)^{-\left(2 A_{t}+1\right) / 2} .
$$

The Student-t distribution extends to infinity in either direction and its moments exist up to order $\ell$ where $\ell<2 A_{t}$ (Kendall and Stuart (1977)). Furthermore, when $k \rightarrow \infty$, and hence $A_{t} \rightarrow \infty$, the Student$\mathrm{t}$ distribution collapses to the Normal distribution. The Student-t distribution has the property that its moment generating function $\int_{-\infty}^{+\infty} e^{\lambda X_{t+1}} g\left(X_{t+1}\right) d X_{t+1}$ does not exist. Unfortunately, this means that the expectation of the marginal rate of substitution, $E_{t}\left(\beta e^{-\alpha X_{t+1}}\right)$, taken under the subjective distribution of future consumption growth rate, is not finite in the Weitzman (2007) economy.

Equations (15) and (16) for the predictive Student-t distribution reiterate equation (24) in Weitzman (2007) in the case of long past history, except the number of degrees of freedom is $k-1$, as also in Shephard (1994), instead of $k$. That is, conditional on the knowledge of large history of consumption growth, $2 A_{t} \approx$ $k-1$ and $B_{t} A_{t}^{-1} \approx V_{t}$ from (12) and therefore $Y_{t+1} \sim \mathrm{t}(k-1)$.

The hallmark of the Weitzman (2007) approach is that it links subjective expectations and learning considerations to asset pricing quantities. In an important result, he shows that the gross riskfree return, denoted $R_{t+1}^{f}$, expressed in log form is (see equation (16) in Weitzman (2007)),

$$
\ln \left(R_{t+1}^{f}\right)=\ln \left(\frac{1}{\beta E_{t}\left(\left(\frac{C_{t+1}}{C_{t}}\right)^{-\alpha}\right)}\right)=-\ln \beta-\ln \underbrace{E_{t}\left(e^{-\alpha X_{t+1}}\right)}_{\rightarrow+\infty} .
$$

Thus, economies that give rise to a predictive Student-t distribution for consumption growth imply that $\ln \left(R_{t+1}^{f}\right) \rightarrow-\infty$, as the expectation of the marginal rate of substitution is not finite. According to Weitzman (2007) such a result constitutes an antipuzzle, since classical models have difficulty explaining the $1 \%$ 
riskfree return (e.g., Mehra and Prescott (1985), Rietz (1988), Weil (1989), and Constantinides (1990)).

Based on his Theorem 1 and 2 and equation (17), the theory of Weitzman (2007) under structural uncertainty and subjective expectations predicts a large equity risk premium, which can be $+\infty$ :

$$
\ln \left(E_{t} R_{t+1}^{e}\right)-\log \left(R_{t+1}^{f}\right)=\ln \left(\frac{E_{t}\left(e^{X_{t+1}}\right)}{\beta E_{t}\left(e^{(1-\alpha) X_{t+1}}\right)}\right)-\ln \left(\frac{1}{\beta E_{t}\left(e^{\left.-\alpha X_{t+1}\right)}\right.}\right) \rightarrow+\infty
$$

Deducing a large equity premium under the predictive Student-t distribution runs counter to the notion that the observed average equity premium of $6 \%$ is too high (Kocherlakota (1996), Mehra and Prescott (2003), and Mehra and Prescott (2008) and references therein). ${ }^{2}$

As Weitzman (2007, p. 1112) acknowledges, the predictive Student-t distribution for consumption growth has undesirable features. For one, the model does not guarantee finite expected utility. Furthermore, the marginal rate of substitution has $E_{t}\left(e^{-\alpha X_{t+1}}\right)=+\infty$ and thus violates Assumption 1 in Hansen and Jagannathan (1991). In addition, the risk-neutral density given by $g\left(Y_{t+1}\right) e^{-\alpha X_{t+1}} / E_{t}\left(e^{-\alpha X_{t+1}}\right)$ (e.g., Harrison and Kreps (1979)), which is central to pricing contingent claims, is not well-defined.

To keep expected utility finite, Weitzman (2007) proposes, but does not formalize, restricting structural uncertainty by confining consumption growth precision between some minimum and maximum levels:

$$
\theta_{t} \in[\underline{\theta}, \bar{\theta}], \quad \text { for all } \mathrm{t}, \quad \underline{\theta}>0,
$$

which imposes a realistic support both for the prior and the posterior for precision given $X^{t}$. Going beyond Weitzman (2007), our incremental contribution is two-fold. First, while imposing a compact support on precision away from zero for all $t$, we (i) establish the finiteness of, and (ii) provide an expression for the moment generating function of consumption growth under structural uncertainty and Bayesian learning. To be able to link asset pricing quantities to risk aversion and structural uncertainty is the impetus for ensuring the finiteness of the moment generating function. Second, exploiting the operational version of

\footnotetext{
${ }^{2}$ The thrust of the theory in Weitzman (2007) is that whenever the agent encounters dynamically evolving structural uncertainty of the type specified in $(6)$, with support $\theta \in(0, \infty)$, the riskfree return can approach minus infinity and the equity premium can approach positive infinity. The predictive Student-t distribution for consumption growth embodies the argument that extreme downside events, even though rare, are so unpalatable that the agent drives down the return on safe bonds but simultaneously forces a high required return on equity.
} 
the theory developed here, we are able to compute equity premium and riskfree return through the moment generating function, a feature central to quantitative assessments. Such computations are not feasible in Weitzman (2007) due to the lack of finiteness of the moment generating function associated with the Student-t distribution.

\section{A Structural Uncertainty Asset Pricing Model under Bounded Volatility}

This section presents the theoretical results used to address whether subjective expectations can help explain asset pricing puzzles. In Theorem 1, we operationalize the restriction that precision have compact support away from zero within a Bayesian learning framework and, in a key departure from Weitzman (2007), we develop the associated posterior density for the precision of consumption growth. Under this modification, Theorem 2 provides a closed-form expression for the predictive density of consumption growth. Furthermore, the moment generating function of consumption growth is derived in Theorem 3.

\section{A. Posterior Density for Precision in the Truncated Gamma Class: A New Conjugacy Result}

To proceed, define $\vartheta_{t}$ to be the precision of consumption growth with finite support denoted by $[\underline{\vartheta}, \bar{\vartheta}]$ with $\underline{\vartheta}>0$. Bear in mind that $\vartheta_{t}$ is not to be confused with $\theta_{t}$, as used in Shephard (1994) and Weitzman (2007) (see our equation (7)), since the latter follows a Gamma distribution and so its support is the entire positive real line. In other words, $\vartheta_{t}$ is the analogue to $\theta_{t}$ under the assumption of bounded support away from zero.

Under the following two assumptions, we establish the conjugacy of $\vartheta_{t+1}$ and $\vartheta_{t}$ in the class of doublytruncated Gamma distributions with support $[\underline{\vartheta}, \bar{\vartheta}]$ for all $t$.

Assumption 3' For a given $t$, the conditional distribution of precision $\vartheta_{t}$ given $X^{t-1}$ is doubly-truncated Gamma with parameters $A_{t-1}$ and $B_{t-1}$ and truncation points $\underline{\vartheta}$ and $\bar{\vartheta}$, denoted by $T G\left(A_{t-1}, B_{t-1} ; \underline{\vartheta}, \bar{\vartheta}\right)$, is given by:

$$
p\left(\vartheta_{t} \mid X^{t-1}\right) \propto \vartheta_{t}^{A_{t-1}-1} e^{-B_{t-1} \vartheta_{t}} \mathbb{I}_{[\underline{\vartheta}, \bar{\vartheta}]}\left(\vartheta_{t}\right)
$$


where the support of the precision distribution is $[\underline{\vartheta}, \vartheta]$ and $\mathbb{I}_{[., .]}(\vartheta)$ denotes an indicator function.

Assumption 4' For a given $t$, the transition equation for precision $\vartheta_{t+1}$ is,

$$
\vartheta_{t+1}=\frac{1}{\omega} \delta_{t+1} \vartheta_{t}, \quad \vartheta_{t} \in[\underline{\vartheta}, \bar{\vartheta}], \quad 0<\omega<1
$$

The conditional distribution of the multiplicative shock $\delta_{t+1}$, given $\vartheta_{t}$ and the history $X^{t}$, is specified in equation (A2) of the Appendix.

Theorem 1 Suppose that, for a given t, (i) Assumption 3' regarding precision $\vartheta_{t}$ and (ii) Assumption 4' regarding multiplicative shock $\delta_{t+1}$ are satisfied. Then, the posterior distribution of $\vartheta_{t+1}$ given $X^{t}$ is also $T G\left(A_{t}, B_{t} ; \underline{\vartheta}, \bar{\vartheta}\right)$ where,

$$
A_{t}=\omega\left(A_{t-1}+\frac{1}{2}\right), \quad B_{t}=\omega\left(B_{t-1}+\frac{\left(x_{t}-\mu\right)^{2}}{2}\right) .
$$

Proof: See the Appendix.

What we have derived in Theorem 1 is an exact result on the posterior distribution of precision $\vartheta_{t+1}$ given $X^{t}$, which is doubly-truncated Gamma, with truncation points $\underline{\vartheta}$ and $\bar{\vartheta}$ (see Coffey and Muller (2000)). Having an explicit characterization of the posterior distribution is imperative to developing a predictive distribution of consumption growth. In particular, our approach formalizes a predictive density framework that prevents extreme beliefs about consumption growth volatility to dominate the analysis. Corresponding to the truncation points of consumption growth volatility defined by

$$
\underline{\sigma} \equiv \frac{1}{\sqrt{\vartheta}} \quad \text { and } \quad \bar{\sigma} \equiv \frac{1}{\sqrt{\underline{\vartheta}}}
$$

we have $\sigma_{t} \in[\underline{\sigma}, \bar{\sigma}]$ for all $t$.

In the theoretical model pursued here, Assumptions 3' and 4' replace Assumptions 3 and 4 of Section I. When the support of consumption growth precision is enlarged to accommodate $\underline{\vartheta} \rightarrow 0$ and $\bar{\vartheta} \rightarrow \infty$, we 
revert back to the setting of Weitzman (2007).

From a broader economic perspective, the structure of beliefs posited in (20) and (21) is essential to ensuring finite expected utility. Paramount for asset pricing formulations, the finiteness of the expected utility ensures a well-posed marginal utility function (e.g., Duffie (1992)). Another sensible way to guarantee finite expected utility is to truncate the support of the distribution of consumption growth given precision. This approach is hampered by the lack of a closed-form expression for the predictive density of consumption growth through Bayesian methods.

Theorem 1 shows that when the conditional distribution $\vartheta_{t}$ given $X^{t-1}$ is truncated Gamma, then the conditional distribution of $\vartheta_{t+1}$ given $X^{t}$ is also truncated Gamma. The distribution of the multiplicative shock $\delta_{t+1}$ in (21) must consequently be outside of the beta distribution class which is defined over $(0,1)$, as in Shephard (1994) and Weitzman (2007). Our choice of the multiplicative shock distribution is designed to preserve the conjugacy of the prior and the posterior to be in the truncated Gamma class. As noted in (A2) of the Appendix, $\delta_{t+1}$ is correlated with $\vartheta_{t}$ in our model.

One aspect of the transition dynamics in (21) deserves further discussion. Shephard (1994) argues that when $\theta_{t}$ is Gamma distributed with support $(0, \infty), \theta_{T} \rightarrow 0$ almost surely, as $T \rightarrow \infty$. To circumvent the undesirable feature that consumption growth volatility eventually becomes infinite, he modifies, using an appropriate dampening constant $r_{t}$, the transition equation to $\theta_{t}=e^{r_{t}} \eta_{t} \theta_{t-1}$ to prevent $\theta_{t}$ from reaching zero. By following the suggestion of Weitzman (2007) and restricting precision $\vartheta_{t}$ on $[\underline{\vartheta}, \bar{\vartheta}]$, we prevent consumption growth volatility from becoming arbitrarily large as the economy evolves. Although not reported, our simulations suggest that for plausible values of $\omega$, it takes several hundred years for the consumption growth volatility to become large even when precision is not truncated and takes values over $(0, \infty)$. Nevertheless, given the observed consumption growth history, precision paths associated with eventually infinite consumption growth volatility will be ignored by the Bayesian investor in his posterior calculations. For these reasons, we keep matters simple and do not introduce a dampening factor in the transition equation (21).

Equation (22) reveals that the evolution of $A_{t}$ and $B_{t}$ is characterized by the same recursion as in Weitzman (2007), where the precision process takes values over the entire positive real line. Nevertheless, the 
distinction is that, in order to make the theory operational, we propose a precision process that maintains bounded support away from zero which, as we illustrate shortly, is crucial for drawing asset pricing implications. Moving backward recursively and assuming that a long past history of length $\tau$ is available in (22), we arrive at:

$$
A_{t} \approx \frac{k-1}{2}, \quad B_{t} \approx \frac{(k-1) V_{t}}{2}, \quad \text { where } V_{t}=\sum_{j=0}^{t-\tau-1} \frac{1}{k}\left(1-\frac{1}{k}\right)^{j}\left(x_{t-j}-\mu\right)^{2}
$$

With such an understanding, we henceforth set $2 A_{t}=k-1$ and $B_{t} A_{t}^{-1}=V_{t}$ in our implementations.

[Fig. 1 about here.]

Given that the support of the precision process is the bounded interval $[\underline{\vartheta}, \bar{\vartheta}$, with $\underline{\vartheta}>0$, the relevant question to ask is whether we are excluding priors that are economically relevant and whether the proposed truncation points are overly restrictive. To address such a concern, we simulate 10 million draws from the posterior distribution $\theta_{t+1}$ given $X^{t}$, when no truncation is imposed, which is $\operatorname{Gamma}\left(A_{t}, B_{t}\right)$. For this exercise, we set the volatility of consumption growth $\sqrt{V}=2 \%$ and the effective sample size to $k=50$. Thus, $A=(k-1) / 2=24.5$ and $B=(k-1) V / 2=0.0098$. Accordingly, we present in Figure 1 , the distribution of both the precision $\theta_{t+1}$ (see Panel A) and the volatility of consumption growth $\sigma_{t+1}=$ $1 / \sqrt{\theta_{t+1}}$ (see Panel B). The message is that even with 10 million draws, it is not feasible to generate values of $\sigma_{t+1}$ above $4.5 \%$ and $\theta_{t+1}$ below 735 . This is an indication that the two precision distributions, without truncation and with truncation, are virtually indistinguishable. To substantiate the claim from a different angle, we calculate $\operatorname{Prob}\left(\sigma_{t+1} \geq \bar{\sigma}\right)$, for values of $\bar{\sigma}$ ranging from $5 \%$ to $500 \%$, and report the probabilities below:

\begin{tabular}{lrrrrrrr}
\hline $\bar{\sigma}=1 / \sqrt{\underline{\theta}}$ & $5 \%$ & $10 \%$ & $20 \%$ & $50 \%$ & $100 \%$ & $200 \%$ & $500 \%$ \\
\hline$k=50$ & $2.60 \mathrm{E}-12$ & $7.71 \mathrm{E}-26$ & $5.38 \mathrm{E}-45$ & $1.07 \mathrm{E}-59$ & $1.96 \mathrm{E}-74$ & $3.50 \mathrm{E}-89$ & $1.11 \mathrm{E}-108$ \\
$k=100$ & $3.08 \mathrm{E}-23$ & $1.62 \mathrm{E}-50$ & $3.32 \mathrm{E}-89$ & $6.62 \mathrm{E}-119$ & $1.11 \mathrm{E}-148$ & $1.77 \mathrm{E}-178$ & $7.15 \mathrm{E}-218$ \\
\hline
\end{tabular}

With parameters corresponding to historical consumption growth, the calculations show that the probabilities are extremely small and decline rapidly in the tails. With $k=100$, the probabilities $\operatorname{Prob}\left(\sigma_{t+1} \geq \bar{\sigma}\right)$ are 
even smaller. Thus, the priors that are being excluded to operationalize the theory have negligible probability. The overall conclusion is that the posterior distributions, with and without imposing the truncation limits, are impossible to differentiate except in the far extreme tails.

\section{B. Predictive Density of Consumption Growth under Bounded Volatility}

Before providing the analytical form of the predictive density of consumption growth in the next theorem, we invoke the following assumption, which is the analogue to Assumption 2 in Section I.

Assumption 2' Conditional on precision $\vartheta_{t+1}$, the consumption growth, $X_{t+1}$, is Normally distributed with

$X_{t+1} \sim N\left(\mu, \frac{1}{\vartheta_{t+1}}\right)$, where the mean $\mu$ is assumed to be a known constant and the precision $\vartheta_{t+1}$ has support $[\underline{\vartheta}, \bar{\vartheta}]$.

Theorem 2 Under Assumptions 2', 3' and 4', the probability density function of the standardized consumption growth $Y_{t+1}$,

$$
Y_{t+1} \equiv \frac{X_{t+1}-\mu}{\sqrt{B_{t} A_{t}^{-1}}}, \quad Y_{t+1} \in \Re
$$

given the history $X^{t}$, is:

$$
g^{\mathrm{DT}}\left(Y_{t+1} ; v_{t}, \underline{\xi}_{t}, \bar{\xi}_{t}\right)=\frac{\gamma\left[\frac{v_{t}+1}{2}, 0.5 \bar{\xi}_{t}\left(1+\frac{Y_{t+1}^{2}}{v_{t}}\right)\right]-\gamma\left[\frac{v_{t}+1}{2}, 0.5 \underline{\xi}_{t}\left(1+\frac{Y_{t+1}^{2}}{v_{t}}\right)\right]}{\sqrt{\pi v_{t}}\left(\gamma\left[v_{t} / 2, \bar{\xi}_{t} / 2\right]-\gamma\left[v_{t} / 2, \underline{\xi}_{t} / 2\right]\right)\left(1+\frac{Y_{t+1}^{2}}{v_{t}}\right)^{\frac{v_{t}+1}{2}}}
$$

where,

$$
\mathrm{v}_{t}=2 A_{t}
$$

represents the degrees of freedom and,

$$
\underline{\xi}_{t}=2 \underline{\vartheta} B_{t}, \quad \bar{\xi}_{t}=2 \bar{\vartheta} B_{t},
$$

represent the truncation parameters. $\gamma[n, j]=\int_{0}^{j} u^{n-1} e^{-u} d u$ is the lower incomplete Gamma function for 
$n>0$. For reasons not yet articulated we call the predictive density in (26), the dampened t distribution.

Proof: See the Appendix.

Suppressing the time subscripts for brevity, notice that when $\underline{\xi} \rightarrow 0$ and $\bar{\xi} \rightarrow \infty$, the density $g^{\mathrm{DT}}(Y ; v, \underline{\xi}, \bar{\xi})$ approaches the Student-t density with $v$ degrees of freedom. The Normal distribution, is obtained as a limiting case when $\underline{\xi} \uparrow \xi$ and $\bar{\xi} \downarrow \xi$ with $\xi>0$. Formally, let $\underline{\xi}=\xi-\varepsilon$ and $\bar{\xi}=\xi+\varepsilon$ and consider the limit of $g^{\mathrm{DT}}\left(Y_{t+1} ; v, \underline{\xi}, \bar{\xi}\right)$ as $\varepsilon \downarrow 0$. Evaluate the following limit,

$$
\begin{aligned}
& \lim _{\underline{\xi} \xi \bar{\xi}, \xi \xi} \frac{\gamma\left[\frac{v+1}{2}, 0.5 \bar{\xi}\left(1+\frac{Y^{2}}{v}\right)\right]-\gamma\left[\frac{v+1}{2}, 0.5 \underline{\xi}\left(1+\frac{Y^{2}}{v}\right)\right]}{\gamma[v / 2, \bar{\xi} / 2]-\gamma[v / 2, \underline{\xi} / 2]} \\
& =\lim _{\varepsilon \downarrow 0} \frac{\frac{1}{\varepsilon}\left(\gamma\left[\frac{v+1}{2}, \frac{\xi+\varepsilon}{2}\left(1+\frac{Y^{2}}{v}\right)\right]-\gamma\left[\frac{v+1}{2}, \frac{\xi-\varepsilon}{2}\left(1+\frac{Y^{2}}{v}\right)\right]\right)}{\frac{1}{\varepsilon}\left(\gamma\left[\frac{v}{2}, \frac{\xi+\varepsilon}{2}\right]-\gamma\left[\frac{v}{2}, \frac{\xi-\varepsilon}{2}\right]\right)} \\
& =\frac{e^{-\frac{\xi}{2}\left(1+\frac{Y^{2}}{v}\right)}\left(\frac{\xi}{2}\left(1+\frac{Y^{2}}{v}\right)\right)^{\frac{v+1}{2}-1}\left(1+\frac{Y^{2}}{v}\right)}{e^{-\frac{\xi}{2}\left(\frac{\xi}{2}\right)^{\frac{v}{2}-1}}}=\sqrt{\frac{\xi}{2}} e^{-\frac{\xi}{2} \frac{Y^{2}}{v}}\left(1+Y^{2} / v\right)^{\frac{v+1}{2}}
\end{aligned}
$$

where the last inequality follows from applying the derivative of the lower incomplete Gamma function $\gamma[n, j]$. Therefore,

$$
\lim _{\underline{\xi} \uparrow, \bar{\xi} \backslash \xi} g^{\mathrm{DT}}\left(Y_{t+1} ; v, \underline{\xi}, \bar{\xi}\right)=\frac{1}{\sqrt{2 \pi(v / \xi)}} e^{-\frac{Y_{t+1}^{2}}{2(v / \xi)}},
$$

which corresponds to the density of the Normal distribution with mean 0 and variance $\frac{v}{\xi}$.

Analogous to the parameterizations invoked by Weitzman (2007), two convenient parameterizations of $g^{\mathrm{DT}}\left(Y_{t+1} ; v, \underline{\xi}, \bar{\xi}\right)$ can be obtained by defining,

$$
\ln \underline{\xi}=\ln v-\frac{1}{I}, \quad \ln \bar{\xi}=\ln v+\frac{1}{I}
$$

where $I$ is a positive constant.

Case 1 Based on the mapping (30), when $I \rightarrow 0$, we have $\underline{\xi} \rightarrow 0$ and $\bar{\xi} \rightarrow \infty$. Accordingly, as $I \rightarrow 0$ the distribution in (26) approaches the Student-t distribution (Geweke (2001) and Weitzman (2007)). 
Case 2 Correspondingly, from (30), as $I \rightarrow \infty$ it follows that $\underline{\xi} \uparrow \vee$ and $\bar{\xi} \downarrow v$. Thus, as $I \rightarrow \infty$ the distribution in (26) converges to the standard Normal distribution.

Case 3 Imposing $2 A_{t}=k-1$ and $B_{t} A_{t}^{-1}=V_{t}$ in (26) of Theorem 2 yields:

$$
g^{\mathrm{DT}}\left(Y_{t+1} ; k-1, \underline{\vartheta}(k-1) V_{t}, \bar{\vartheta}(k-1) V_{t}\right)
$$

which corresponds to the case of a large number of past observations on consumption growth from (24).

Our characterizations imply that the three-parameter density family $g^{\mathrm{DT}}\left(Y_{t+1} ; v_{t}, \underline{\xi}_{t}, \bar{\xi}_{t}\right)$ lies in between the Normal distribution and the Student-t distribution. But, is the predictive density (26) closer to the heavy-tailed Student-t distribution (14) or the thin-tailed Normal distribution? A parsimonious way to capture the difference between (14) and (26) is to compute the logarithm of the ratio of the two densities when a large past history of consumption growth is available (i.e., from Case $3,2 A_{t}=k-1, B_{t} A_{t}^{-1}=V_{t}$ ):

$$
\Upsilon_{t+1} \equiv \ln \left(\frac{g\left(X_{t+1} ; k-1\right)}{g^{\mathrm{DT}}\left(X_{t+1} ; k-1, \underline{\vartheta}(k-1) V_{t}, \bar{\vartheta}(k-1) V_{t}\right)}\right), \quad X_{t+1}=\mu+\sqrt{V_{t}} Y_{t+1}
$$

Figure 2 plots $\Upsilon_{t+1}$ versus consumption growth $X_{t+1}$ taking $\mu=2 \%, \sqrt{V}=2 \%, k=50, \underline{\sigma}=1 / \sqrt{\bar{\vartheta}}=0.01 \%$ and $\bar{\sigma}=1 / \sqrt{\underline{\vartheta}}=500 \%$. The flat region in the bowl-shaped curve has the interpretation that the two predictive densities are equivalent over consumption growth of roughly $\pm 1,700 \%$. Even when differences are observed in the tails, the distinction is of the order of $10^{-7}$. Intuitively, this slight departure occurs as the Student-t distribution has raw moments up to order $\ell<k-1$, whereas the density in (26) has bounded algebraic moments of all orders (shown next in equation (36) of Theorem 3). Given the inherent closeness of the two predictive distributions, the density in (26), is labeled as the dampened t distribution. The source of the dampening is the difference between the incomplete Gamma function evaluated at the upper and the lower truncation points, as seen from (26). It is the incomplete Gamma trimming that ensures finite moment generating function. ${ }^{3}$

\footnotetext{
${ }^{3}$ Shephard (1994) has also considered generalized error distribution as the building block for the conditional distribution of $X_{t} \mid \vartheta_{t}$. Such a treatment along with a generalized Gamma distributed precision process induces a predictive distribution that is generalized Student-t. However, the moment generating function still does not exist and therefore hinders the usefulness of
} 
[Fig. 2 about here.]

There is an alternative way to construct the predictive density (26). Suppose $Z$ is a standard Normal variate and $W$ follows a $\chi^{2}$ distribution with $v$ degrees of freedom truncated on the interval $[\underline{\xi}, \bar{\xi}]$ where $0<\underline{\xi}<\bar{\xi}<+\infty$, then it is shown via Lemma 2 in the Appendix that $\frac{Z}{\sqrt{W / v}} \equiv Y$ obeys the dampened $\mathrm{t}$ distribution with density (26). It is this construction that we exploit to analytically derive the moment generating function of $Y_{t+1}$ and show that it exists and is well-defined.

\section{Finiteness of the Moment Generating Function of Consumption Growth}

Our innovation is that asset pricing quantities can be evaluated using the moment generating function of $g^{\mathrm{DT}}\left(Y_{t+1} ; v_{t}, \underline{\xi}_{t}, \bar{\xi}_{t}\right)$, a task that cannot be accomplished under the Student-t distribution. Suppressing time subscripts on $v_{t}, \underline{\xi}_{t}, \bar{\xi}_{t}, A_{t}$ and $B_{t}$ we now state the next important result.

Theorem 3 For the predictive density of $Y_{t+1}=\frac{X_{t+1}-\mu}{\sqrt{B A^{-1}}}$ specified in (26), the moment generating function is:

$$
\begin{aligned}
\Psi_{Y}[\lambda] & \equiv E_{t}\left(e^{\lambda Y_{t+1}}\right)=\int_{-\infty}^{+\infty} e^{\lambda Y_{t+1}} g^{\mathrm{DT}}\left(Y_{t+1} ; \nu, \underline{\xi}, \bar{\xi}\right) d Y_{t+1} \\
& =\frac{1}{c[v, \underline{\xi}, \bar{\xi}]} \int_{\underline{\xi}}^{\bar{\xi}} e^{\frac{\lambda^{2} v}{2 w}} w^{\frac{v}{2}-1} e^{-\frac{w}{2}} d w
\end{aligned}
$$

where $c[v, \underline{\xi}, \bar{\xi}]=2^{v / 2}(\gamma[v / 2, \bar{\xi} / 2]-\gamma[v / 2, \underline{\xi} / 2]), v=2 A$, and $\underline{\xi}=2 \underline{\vartheta} B$ and $\bar{\xi}=2 \bar{\vartheta} B$. The moment generating function of consumption growth $X_{t+1}$ is then,

$$
\Psi_{X}[\lambda]=e^{\mu \lambda} \Psi_{Y}\left[\lambda \sqrt{B A^{-1}}\right]
$$

since $X_{t+1}=\mu+\sqrt{B A^{-1}} Y_{t+1}$. All odd-order moments of $Y_{t+1}$ are equal to 0 . The even-order moments of

the model for examining asset pricing puzzles. To our knowledge, when (i) $X_{t} \mid \vartheta_{t}$ follows generalized error distribution, and (ii) precision process follows (21) and lives on a compact support away from zero, the predictive distribution is unamenable to analytical characterization through Bayesian methods. 
$Y_{t+1}$ are given by,

$$
E_{t}\left(Y_{t+1}^{2 \phi}\right)=\frac{(2 \phi) !}{\phi !}\left(\frac{v}{2}\right)^{\phi} \frac{1}{c[v, \underline{\xi}, \bar{\xi}]} \int_{\underline{\xi}}^{\bar{\xi}} w^{\frac{v}{2}-\phi-1} e^{-\frac{w}{2}} d w<\infty
$$

for $\phi=1,2, \ldots, \infty$.

Proof: See the Appendix.

Unlike the Student-t distribution, which does not possess a finite moment generating function, it is established that the dampened $\mathrm{t}$ distribution has a finite moment generating function for all $0<\underline{\xi}<\bar{\xi}<+\infty$. At the crux of Theorem 3 is the statement that the moment generating function is the integral of a continuous function over a compact support, and consequently finite (e.g., Lukacs (1960)). Well-defined preferences and marginal rate of substitution in the economy hinge on the finiteness of the moment generating function $\Psi_{X}[\lambda]$.

\section{Can Structural Uncertainty Resolve Asset Pricing Puzzles?}

Under structural parameter uncertainty, where the consumption growth volatility $\sigma_{t}$ has support $[\underline{\sigma}, \bar{\sigma}]$, and Bayesian learning, the operational version of theory shows that the primitive distribution for consumption growth, which is Normal, gets transformed to the heavy-tailed dampened t distribution. Structural parameter uncertainty induced tail thickening of the predictive consumption growth distribution can, in principle, exert a substantial impact on asset pricing quantities through the marginal rate of substitution.

To see the restrictions imposed by the present theory, we note that the riskfree return and the equity risk premium can both be obtained from the moment generating function of consumption growth, $\Psi_{X}[\lambda]$. Guided by equations (17) and (18), we obtain:

$$
\begin{aligned}
\ln \left(R_{t+1}^{f}\right) & =-\ln \beta-\ln \Psi_{X}[-\alpha] \\
\ln \left(E_{t} R_{t+1}^{e}\right)-\ln \left(R_{t+1}^{f}\right) & =\ln \Psi_{X}[1]-\ln \Psi_{X}[1-\alpha]+\ln \Psi_{X}[-\alpha]
\end{aligned}
$$

where $\Psi_{X}[\lambda]$ is presented in equation (35) of Theorem 3. The special feature of this economy with subjec- 
tive expectations is that it supports a finite moment generating function of $X_{t+1}$. Therefore, the contribution of our approach lies in that asset pricing quantities are computable, which is essential for conducting quantitative analysis.

Economic theory now suggests that the equity risk premium and the riskfree return are both linked to the parameters of the dampened t distribution. In particular, the asset pricing quantities are restricted by $\left(A_{t}, B_{t}, \underline{\xi}_{t}, \bar{\xi}_{t}\right)$, which jointly control the mean, the volatility, and the truncation points of the belief process. Accounting for subjective expectations expands the traditional consumption-based asset pricing model.

The tractability of our constructions allows us to pose and answer outstanding questions of broad interest: (1) What is the quantitative impact of subjective expectations on asset pricing? (2) What level of structural uncertainty is required for resolving asset pricing puzzles? and (3) In what sense does structural uncertainty quantitatively map into consumption disaster fears? Operationalizing the theory, as established in Theorems 1 through 3, enables us to address these questions in the ensuing subsections, a task not feasible in the environment of Weitzman (2007).

\section{A. Plausible Structural Uncertainty Does not Profoundly Affect Asset Pricing Quantities}

In our quantitative assessments, we rely on the assumption of sufficiently many past observations which yields $2 A_{t}=k-1$ and $B_{t} A_{t}^{-1}=V_{t}$. We use benchmark values that are in line with the existing literature (e.g., Mehra and Prescott (1985), Barro (2006), and Weitzman (2007)):

$$
\mu=2 \%, \quad \sqrt{V}=2 \%, \quad \beta=0.99, \quad k \in\{10,30,50\}, \quad \underline{\sigma}=1 / \sqrt{\bar{\vartheta}}=0.1 \% .
$$

The lower bound on structural uncertainty $\underline{\sigma}=1 / \sqrt{\bar{\vartheta}}$ is not essential and therefore held fixed. The effective sample size $k$ is selected to cover a range of reasonable values. In terms of subjective expectations, $k$ measures how much confidence the investor places in the variance estimate, and at the same time it controls the degrees of freedom, and therefore the tail thickness, of the predictive distribution of consumption growth.

Table 1 first assesses the quantitative impact of subjective expectations on $\ln R_{t+1}^{f}$ and $\ln \left(E_{t} R_{t+1}^{e}\right)-$ 
$\ln \left(R_{t+1}^{f}\right)$ by adopting risk aversion $\alpha \in\{2,3,5,10\}$. Considering that the average historical volatility of consumption growth is $2 \%$, the maximum allowable level of consumption growth volatility is fixed at $\bar{\sigma}=1 / \sqrt{\underline{\vartheta}}=50 \%$.

Observe that the effect of subjective expectations on asset pricing quantities is barely noticeable over reasonable levels of $\underline{\sigma}, \bar{\sigma}$, and $\alpha$. We draw this conclusion from three angles. First, the riskfree return and the equity premium are almost identical between the case $k=50$ and the full information Normal distribution benchmark. Second, lower levels of $k$ tends to decrease both the riskfree return and the equity return, more strongly so for the riskfree return. However, the strength of the variation of asset pricing quantities with respect to $k$ is dependent on $\alpha$ : weaker at $\alpha=\{2,3,5\}$ but pronounced at $\alpha=10$. Third, and more importantly, the maximum equity premium the model is able to generate is $1.70 \%$ with $k=10$ and $\alpha=10$.

We also find, but not report, that the response of the riskfree return and the equity premium to $\bar{\sigma}$ is virtually flat up to $\bar{\sigma}=190 \%$ for $\alpha \in(2,30)$ with $k=50$, which we consider to be a reasonable candidate for the effective sample size. To save on space, all results are henceforth reported for $k=50$.

[Fig. 3 about here.]

On the other hand, when excessive levels of structural uncertainty are permitted to influence asset pricing, $\ln R_{t+1}^{f}$ and $\ln \left(E_{t} R_{t+1}^{e}\right)-\ln \left(R_{t+1}^{f}\right)$ rise rapidly with small shifts in $\bar{\sigma}$ as seen from Figure 3. Possibly rectifying the shortcomings of the consumption-based asset pricing model, the presence of structural uncertainty now sharply lowers the riskfree return and raises the equity risk premium. Consider $\alpha=3$ in Figure 3 to illustrate the main ideas. At a value of $\bar{\sigma}=762.50 \%, \ln R_{t+1}^{f}=5.65 \%$ and $\ln \left(E_{t} R_{t+1}^{e}\right)-\ln \left(R_{t+1}^{f}\right)=1.28 \%$ while at $\bar{\sigma}=766.50 \%$ the counterpart values are $-6.30 \%$ and $13.24 \%$. The model behavior is knife-edged sensitive at large levels of structural uncertainty regardless of the magnitude of risk aversion.

In summary, Table 1 and Figure 3 together give rise to two inferences about the implications of subjective expectations for asset pricing. First, the particularly parameterized structural uncertainty model operationalized here does not produce a significant change in the asset pricing quantities unless excessive a priori uncertainty is entertained. Second, it points to the fragility of the predictive Student-t distribution for 
consumption growth where $\ln R_{t+1}^{f} \rightarrow-\infty$ and $\ln \left(E_{t} R_{t+1}^{e}\right)-\ln \left(R_{t+1}^{f}\right) \rightarrow+\infty$. That is, when the distribution of $\sigma_{t}$, or equivalently precision $\vartheta_{t}$, is restricted over a compact support away from zero, the minimum generated riskfree return and the maximum generated equity premium are both finite. Nonetheless, the magnitudes fall short of the historical counterparts even though the dampened t distribution is virtually indistinguishable from the Student-t distribution. The conclusions drawn here are robust even when consumption growth volatility is increased to $\sqrt{V}=3.5 \%$ as in Barro (2006). For instance, when $k=10$ and $\alpha=10$, the model equity premium is $1.78 \%$ and the riskfree return is $14.02 \%$.

\section{B. It is Still Challenging to Explain the Equity Premium of 6\% and the Riskfree Return of $1 \%$}

Results presented in Table 2 are at the heart of asset pricing puzzles. Focus first on Panel A, where we numerically search over the maximum level of consumption growth volatility, $\bar{\sigma}$, required to match the $6 \%$ average equity risk premium. When $\alpha$ is restricted between 2 and 10, the range of $\bar{\sigma}$ required to match the equity risk premium varies between $199.26 \%$ and $1,193.50 \%$. Most financial economists hold the view that $\alpha$ should lie somewhere between 2 and 10 (e.g., Mehra and Prescott (1985) and Kocherlakota (1996)).

The inability of the model to match economic theory with data is also implicit in the corresponding values of riskfree return when the equity risk premium is matched to $6 \%$. In particular, the simultaneous justification of asset returns data requires levels of $\bar{\sigma}$ between $765.5 \%$ and $1,193.50 \%$ when $\alpha$ is confined between between 2 and 3. In sum, a mismatch still exists when asset pricing theory incorporating subjective expectations is applied to data from financial markets.

Panel B of Table 2 conducts the analog exercise where we search over the maximum level of consumption growth volatility, $\bar{\sigma}$, to match the riskfree return of $1 \%$ for a given $\alpha$. Deficiencies of the model manifest themselves from a different perspective: declining values of $\bar{\sigma}$ that coincide with increasing $\alpha$, also imply increasing equity risk premium in order to maintain the target riskfree return of $1 \%$.

To reiterate, a reasonably parameterized asset pricing model under subjective uncertainty about the volatility of consumption growth faces a formidable hurdle in explaining the equity risk premium and the real riskfree return simultaneously. The level of structural uncertainty required to resolve asset pricing 
puzzles in the model is, in our view, beyond any reasonable norm.

\section{Priors Yielding Similar Disaster Probabilities Have Vastly Different Asset Pricing Implica- tions}

How does the entertained value of $\bar{\sigma}=1 / \sqrt{\underline{\vartheta}}$ translate into probabilities of consumption disasters highlighted in Weitzman (2007)? Consider the possibility of a rare disaster $x^{\text {rare }}$, which represents a deviation from mean consumption growth in multiples of volatility:

$$
x^{\mathrm{rare}}=\mu-h \sqrt{V}
$$

where the multiple $h \in\{3,4,5,6\}, \mu=2 \%$, and $\sqrt{V}=2 \%$. A 5-sigma downside event corresponds to $h=5$ and implies a $8 \%$ drop in consumption.

The additional advantage of our approach is that it is amenable to computing the probability of rare events analytically. It is shown in the Appendix that,

$$
\operatorname{Prob}\left(X_{t+1} \leq x^{\mathrm{rare}}\right)=\operatorname{Prob}\left(Y_{t+1} \leq-h\right)=\frac{1}{c[\mathrm{v}, \underline{\xi}, \bar{\xi}]} \int_{\underline{\xi}}^{\bar{\xi}} N(-h \sqrt{w / v}) w^{\frac{v}{2}-1} e^{-\frac{w}{2}} d w
$$

where $v=k-1$ is the degrees of freedom, $\underline{\xi}=\frac{(k-1) V}{\bar{\sigma}^{2}}, \bar{\xi}=\frac{(k-1) V}{\underline{\sigma}^{2}}$, and $N(d)=\frac{1}{\sqrt{2 \pi}} \int_{-\infty}^{d} e^{-u^{2} / 2} d u$ is the Normal probability integral.

Table 3 presents the probabilities of rare disasters as a function of maximum level of consumption growth volatility. Specifically, we present the probabilities corresponding to the Normal distribution, the dampened t distribution, and the Student-t distribution.

The main message is that the maximum allowable level of consumption growth volatility is not instrumental to the probability of rare disasters in the model. Explaining the equity premium in the model with reasonable $\alpha$ requires a maximum level of consumption growth volatility in excess of $765 \%$ and these implausible prior beliefs have in common disaster probabilities that are indistinguishable from those implied by more realistic priors. 
Note that, at the same time, the two sets of prior beliefs have diametrically opposite asset pricing implications: one asserting, and the other contradicting, the antipuzzle view. Our explicit quantification of the equity premium along with the rare disaster probabilities, possibly provides a reason to challenge the argument offered in Weitzman (2007) that large levels of equity premium are somehow compensation for structural uncertainty and consumption disaster fears.

\section{Conclusions}

In this paper, we contribute to the literature on asset pricing models with Bayesian learning by providing an operational version of the structural uncertainty approach that incorporates a compact support for consumption growth volatility. Our setting incorporates realistic priors about consumption growth volatility and has substantial economic consequences. First, it guarantees finite asset pricing quantities. Second, it allows characterization of the Bayesian predictive density for consumption growth that is virtually indistinguishable from the heavy-tailed Student-t distribution, but importantly possesses a finite moment generating function. The resulting asset pricing model with subjective expectations yields finite expected utility, and tractable expressions for equity premium and riskfree return. In this setting, embedding structural uncertainty induces a heavy-tailed predictive distribution for consumption growth without introducing jumps in the consumption growth process.

The availability of the moment generating function of consumption growth renders quantitative evaluation feasible. Applying economic theory in the context of subjective expectations reveals that explaining the historical equity premium and riskfree return requires implausible levels of uncertainty about consumption growth volatility. Furthermore, these implausible prior beliefs share disaster probabilities that almost coincide with those implied by more realistic priors. However, the two sets of prior beliefs have diametrically opposite asset pricing implications: one asserting, and the other contradicting, the antipuzzle view favored by Weitzman (2007).

The scope of the approach developed here can be expanded along several directions to enhance our understanding of how subjective expectations impact asset prices. First, the asset pricing framework can 
be extended to investigate higher-dimensional structural uncertainty, where the agent faces uncertainty not only about consumption growth volatility, but also about skewness and kurtosis. Second, the effect of more realistic consumption growth dynamics can be explored by accommodating serial correlation in the specification of the consumption growth process (e.g., Barsky and DeLong (1993), Bansal and Yaron (2004), and Tsionas (2005)). Finally, further insights could be gained by considering richer pricing kernel specifications such as those involving habit formation (e.g., Constantinides (1990) and Campbell and Cochrane (1999)). These extensions are left for future work. 


\section{Appendix: Proof of Results}

Distribution of the Multiplicative Shock $\delta_{t+1}$ in (21): What we specify first is the distribution of the multiplicative shock $\delta_{t+1}$ that guarantees the conjugacy of the system while ensuring that the support of precision process $\vartheta_{t}$ remains $[\underline{\vartheta}, \bar{\vartheta}]$ through time.

To appreciate our generalization, let us momentarily return to the Weitzman (2007) model where the precision process $\theta_{t}$ evolves according to the transition equation $\theta_{t+1}=\frac{1}{\omega} \eta_{t+1} \theta_{t}$ as in (6). Importantly, when $\theta_{t}$ satisfies $\theta_{t} \mid X^{t} \sim \operatorname{Gamma}\left(a_{t}, b_{t}\right)$ and the multiplicative shock $\eta_{t+1}$ follows a $\operatorname{Beta}\left(\omega a_{t},(1-\omega) a_{t}\right)$ distribution, then $\theta_{t+1} \mid X^{t} \sim \operatorname{Gamma}\left(A_{t}, B_{t}\right)$ where $A_{t}=\omega a_{t}$ and $B_{t}=\omega b_{t}$. Such a structure preserves congugacy of the system through time.

In order to provide the details of the specification of the multiplicative shock $\delta_{t+1}$ in (21), we introduce additional notation for brevity of presentation. Conditionally on $X^{t}$, let $f_{t}(\cdot)$ and $F_{t}(\cdot)$ denote the pdf and cdf of $\theta_{t}$, respectively. Similarly, given $X^{t}$, let $g_{t}(\cdot)$ and $G_{t}(\cdot)$ denote the pdf and cdf of $\theta_{t+1}$, respectively. That is, $f_{t}(\cdot)$ is the $\operatorname{Gamma}\left(a_{t}, b_{t}\right)$ density and $g_{t}(\cdot)$ is the Gamma $\left(\omega a_{t}, \omega b_{t}\right)$ density. Furthermore, let $h_{t}\left(\theta_{t}, \theta_{t+1}\right)$ denote the joint pdf of $\left(\theta_{t}, \theta_{t+1}\right)$, conditionally on $X^{t}$. This implies that the two marginal distributions associated with the joint density $h_{t}$ are $f_{t}$ and $g_{t}$, respectively.

Now denote by $\mathfrak{f}_{t}$ and $\mathfrak{F}_{t}$ the pdf and cdf of the truncated Gamma $\operatorname{TG}\left(a_{t}, b_{t} ; \underline{\vartheta}, \bar{\vartheta}\right)$ distribution, respectively. Furthermore, denote by $\mathfrak{g}_{t}$ and $\mathfrak{G}_{t}$ the pdf and cdf of the truncated Gamma TG $\left(\omega a_{t}, \omega b_{t} ; \underline{\vartheta}, \bar{\vartheta}\right)$ distribution, respectively. When $\underline{\vartheta} \rightarrow 0$ and $\bar{\vartheta} \rightarrow \infty$, the TG density $\mathfrak{f}_{t}$ converges to the Gamma density $f_{t}$, and the TG density $\mathfrak{g}_{t}$ converges to the Gamma density $g_{t}$.

The distribution of the multiplicative shock of $\delta_{t+1}$ is then defined as follows. Given $\vartheta_{t}$ and $X^{t}$, the conditional pdf of $\delta_{t+1}$ is:

$$
p\left(\delta_{t+1} \mid \vartheta_{t}, X^{t}\right)=\frac{\vartheta_{t}}{\omega} \cdot \mathfrak{g}_{t}\left(\frac{1}{\omega} \vartheta_{t} \delta_{t+1}\right) \cdot \frac{h_{t}\left(F_{t}^{-1}\left(\mathfrak{F}_{t}\left(\vartheta_{t}\right)\right), G_{t}^{-1}\left(\mathfrak{G}_{t}\left(\frac{1}{\omega} \vartheta_{t} \boldsymbol{\delta}_{t+1}\right)\right)\right)}{f_{t}\left(F_{t}^{-1}\left(\mathfrak{F}_{t}\left(\vartheta_{t}\right)\right)\right) \cdot g_{t}\left(G_{t}^{-1}\left(\mathfrak{G}_{t}\left(\frac{1}{\omega} \vartheta_{t} \delta_{t+1}\right)\right)\right)}
$$

While equation (A2) might seem complicated, it is a generalization of the Beta distribution used for the multiplicative shock in the case of the (untruncated) Gamma precision process $\theta_{t}$. Indeed, in the limit 
$\underline{\vartheta} \rightarrow 0$ and $\bar{\vartheta} \rightarrow \infty$, the conditional density (A2) converges to the Beta $\left(\omega a_{t},(1-\omega) a_{t}\right)$ distribution, namely the distribution of the multiplicative shock $\eta_{t+1}$ in Weitzman (2007). To establish this property, we build on the following Lemma:

Lemma 1 The joint $p d f h_{t}(\cdot, \cdot)$ of $\left(\theta_{t}, \theta_{t+1}\right)$, conditionally on $X^{t}$, is given by

$$
h_{t}\left(\theta_{t}, \theta_{t+1}\right)=\frac{\omega}{\Gamma\left[\omega a_{t}\right] \Gamma\left[(1-\omega) a_{t}\right]} b_{t}^{a_{t}} e^{-b_{t} \theta_{t}} \cdot\left(\omega \theta_{t+1}\right)^{\omega a_{t}-1}\left(\theta_{t}-\omega \theta_{t+1}\right)^{(1-\omega) a_{t}-1},
$$

where $0<\theta_{t+1}<\frac{\theta_{t}}{\omega}$.

Proof of Lemma 1. The pdf of $\theta_{t}$, given $X^{t}$, is the Gamma $\left(a_{t}, b_{t}\right)$ density, i.e., $p\left(\theta_{t} \mid X^{t}\right)=\frac{b_{t}^{a_{t}}}{\Gamma\left[a_{t}\right]} \theta_{t}^{a_{t}-1} e^{-b_{t} \theta_{t}}$. Since $\theta_{t+1}=\frac{1}{\omega} \eta_{t+1} \theta_{t}$ and $\eta_{t+1} \sim \operatorname{Beta}\left(\omega a_{t},(1-\omega) a_{t}\right)$ with density $k_{t}(\eta)=\frac{1}{B\left[\omega a_{t},(1-\omega) a_{t}\right]} \eta^{\omega a_{t}-1}(1-\eta)^{(1-\omega) a_{t}-1}$, it follows that:

$$
p\left(\theta_{t+1} \mid \theta_{t}, X^{t}\right)=\frac{\omega}{\theta_{t}} k_{t}\left(\frac{\omega}{\theta_{t}} \theta_{t+1}\right)=\frac{\omega}{\theta_{t}} \frac{1}{B\left[\omega a_{t},(1-\omega) a_{t}\right]}\left(\frac{\omega}{\theta_{t}} \theta_{t+1}\right)^{\omega a_{t}-1}\left(1-\frac{\omega}{\theta_{t}} \theta_{t+1}\right)^{(1-\omega) a_{t}-1} .
$$

By noting that $h_{t}\left(\theta_{t}, \theta_{t+1}\right)=p\left(\theta_{t}, \theta_{t+1} \mid X^{t}\right)=p\left(\theta_{t+1} \mid \theta_{t}, X^{t}\right) p\left(\theta_{t} \mid X^{t}\right)$ proves the result.

In the limiting case with $\underline{\vartheta} \rightarrow 0$ and $\bar{\vartheta} \rightarrow \infty$, we have $\mathfrak{f}_{t} \rightarrow f_{t}, \mathfrak{F}_{t} \rightarrow F_{t}$, and $\mathfrak{g}_{t} \rightarrow g_{t}, \mathfrak{G}_{t} \rightarrow G_{t}$. Hence, the density $p\left(\delta_{t+1} \mid \vartheta_{t}\right)$ converges to $\frac{\vartheta_{t}}{\omega} \frac{h_{t}\left(\vartheta_{t} \frac{1}{\omega} \vartheta_{t} \delta_{t+1}\right)}{f_{t}\left(\vartheta_{t}\right)}$. The density $f_{t}\left(\vartheta_{t}\right)$ is given by $f_{t}\left(\vartheta_{t}\right)=\frac{b_{t}^{a_{t}}}{\Gamma\left(a_{t}\right)} \vartheta_{t}^{a_{t}-1} e^{-b_{t} \vartheta_{t}}$ and Lemma 1 implies that

$$
h_{t}\left(\vartheta_{t}, \frac{1}{\omega} \vartheta_{t} \delta_{t+1}\right)=\frac{\omega}{\Gamma\left[\omega a_{t}\right] \Gamma\left[(1-\omega) a_{t}\right]} b_{t}^{a_{t}} \vartheta_{t}^{a_{t}-2} e^{-b_{t} \vartheta_{t}} \cdot \delta_{t+1}^{\omega a_{t}-1}\left(1-\delta_{t+1}\right)^{(1-\omega) a_{t}-1}
$$

Thus, as $\underline{\vartheta} \rightarrow 0$ and $\bar{\vartheta} \rightarrow \infty$, the limit of $p\left(\delta_{t+1} \mid \vartheta_{t}\right)$ is:

$$
\frac{\vartheta_{t}}{\omega} \frac{h_{t}\left(\vartheta_{t}, \frac{1}{\omega} \vartheta_{t} \delta_{t+1}\right)}{f_{t}\left(\vartheta_{t}\right)}=\frac{\Gamma\left[a_{t}\right]}{\Gamma\left[\omega a_{t}\right] \Gamma\left[(1-\omega) a_{t}\right]} \delta_{t+1}^{\omega a_{t}-1}\left(1-\delta_{t+1}\right)^{(1-\omega) a_{t}-1}
$$

which is simply the $\operatorname{Beta}\left(\omega a_{t},(1-\omega) a_{t}\right)$ density of the multiplicative shock $\eta_{t+1}$ in (Weitzman 2007). Thus, the choice of $\delta_{t+1}, \vartheta_{t}$ and $\vartheta_{t+1}$ ensures the internal consistency of the system and yet maintains the conjugacy of $\vartheta_{t}$ and $\vartheta_{t+1}$, as verified next. 
Proof of Theorem 1 (Posterior Distribution of $\vartheta_{t+1}$ given $X^{t}$ ): It is shown that the conditional distribution of $\vartheta_{t}$, given $X^{t}$, is $\operatorname{TG}\left(A_{t}, B_{t} ; \underline{\vartheta}, \bar{\vartheta}\right)$ where $A_{t}=\omega a_{t}=\omega\left(A_{t-1}+\frac{1}{2}\right)$ and $B_{t}=\omega b_{t}=\omega\left(B_{t-1}+\frac{\left(X_{t}-\mu\right)^{2}}{2}\right)$.

First, observe that the conditional distribution of $\vartheta_{t}$ given $X^{t}$ is given by

$$
\begin{aligned}
p\left(\vartheta_{t} \mid X^{t}\right) & \propto p\left(X^{t}, \vartheta_{t}\right)=p\left(X_{t} \mid \vartheta_{t}\right) p\left(\vartheta_{t} \mid X^{t-1}\right) \\
& \propto \sqrt{\vartheta_{t}} e^{-\frac{\left(X_{t}-\mu\right)^{2} \vartheta_{t}}{2}} \cdot \vartheta_{t}^{A_{t-1}-1} e^{-B_{t-1} \vartheta_{t}} \mathbb{I}_{[\underline{\vartheta}, \bar{\vartheta}]}\left(\vartheta_{t}\right) \\
& \propto \vartheta_{t}^{a_{t}-1} e^{-b_{t} \vartheta_{t}} \mathbb{I}_{[\underline{\vartheta}, \bar{\vartheta}]}\left(\vartheta_{t}\right)
\end{aligned}
$$

where $a_{t}=A_{t-1}+\frac{1}{2}$ and $b_{t}=B_{t-1}+\frac{\left(X_{t}-\mu\right)^{2}}{2}$. Hence, the conditional distribution of $\vartheta_{t}$ given $X^{t}$ is TG $\left(a_{t}, b_{t} ; \underline{\vartheta}, \bar{\vartheta}\right)$.

In our notation, the density of $\vartheta_{t}$ given $X^{t}$ is $\mathfrak{f}_{t}(\cdot)$. Given the conditional density (A2) of the multiplicative shock $\delta_{t+1}$, it follows that the joint density of $\left(\vartheta_{t}, \delta_{t+1}\right)$, conditionally on $X^{t}$, is

$$
\psi_{t}\left(\vartheta_{t}, \delta_{t+1}\right)=\frac{\vartheta_{t}}{\omega} \cdot \mathfrak{g}_{t}\left(\frac{1}{\omega} \vartheta_{t} \delta_{t+1}\right) \cdot \frac{h_{t}\left(F_{t}^{-1}\left(\mathfrak{F}_{t}\left(\vartheta_{t}\right)\right), G_{t}^{-1}\left(\mathfrak{G}_{t}\left(\frac{1}{\omega} \vartheta_{t} \delta_{t+1}\right)\right)\right)}{f_{t}\left(F_{t}^{-1}\left(\mathfrak{F}_{t}\left(\vartheta_{t}\right)\right)\right) \cdot g_{t}\left(G_{t}^{-1}\left(\mathfrak{G}_{t}\left(\frac{1}{\omega} \vartheta_{t} \delta_{t+1}\right)\right)\right)} \mathfrak{f}_{t}\left(\vartheta_{t}\right)
$$

Based on the posited transition equation of the (truncated) precision process, $\vartheta_{t+1}=\frac{1}{\omega} \delta_{t+1} \vartheta_{t}$ we can now exploit the Jacobian of the appropriate two-dimensional transformation. Specifically, if $(X, Y)$ has joint pdf $f_{X, Y}(x, y)$ and $(W, Z)=\left(X, \frac{1}{c} X Y\right)$, then the joint pdf of $(W, Z)$ is $f_{W, Z}(w, z)=c \frac{1}{w} f_{X, Y}\left(w, c \frac{z}{w}\right)$. Consequently, the joint pdf of $\left(\vartheta_{t}, \vartheta_{t+1}\right)$, conditionally on $X^{t}$, is:

$$
\mathfrak{h}_{t}\left(\vartheta_{t}, \vartheta_{t+1}\right)=\mathfrak{f}_{t}\left(\vartheta_{t}\right) \cdot \mathfrak{g}_{t}\left(\vartheta_{t+1}\right) \cdot \frac{h_{t}\left(F_{t}^{-1}\left(\mathfrak{F}_{t}\left(\vartheta_{t}\right)\right), G_{t}^{-1}\left(\mathfrak{G}_{t}\left(\vartheta_{t+1}\right)\right)\right)}{f_{t}\left(F_{t}^{-1}\left(\mathfrak{F}_{t}\left(\vartheta_{t}\right)\right)\right) \cdot g_{t}\left(G_{t}^{-1}\left(\mathfrak{G}_{t}\left(\vartheta_{t+1}\right)\right)\right)}
$$

The marginal density of $\vartheta_{t+1}$, given $X^{t}$, is then obtained by integrating out $\vartheta_{t}: p\left(\vartheta_{t+1} \mid X^{t}\right)=\int \mathfrak{h}_{t}\left(\vartheta_{t}, \vartheta_{t+1}\right) d \vartheta_{t}$. Using the transformation $\zeta_{t}=F_{t}^{-1}\left(\mathfrak{F}_{t}\left(\vartheta_{t}\right)\right)$, we obtain $F_{t}\left(\zeta_{t}\right)=\mathfrak{F}_{t}\left(\vartheta_{t}\right)$ and then differentiating yields $f_{t}\left(\zeta_{t}\right) d \zeta_{t}=\mathfrak{f}_{t}\left(\vartheta_{t}\right) d \vartheta_{t}$. Hence, the above integral reduces to

$$
\int \mathfrak{h}_{t}\left(\vartheta_{t}, \vartheta_{t+1}\right) d \vartheta_{t}=\frac{\mathfrak{g}_{t}\left(\vartheta_{t+1}\right)}{g_{t}\left(G_{t}^{-1}\left(\mathfrak{G}_{t}\left(\vartheta_{t+1}\right)\right)\right)} \cdot \int h_{t}\left(\zeta_{t}, G_{t}^{-1}\left(\mathfrak{G}_{t}\left(\vartheta_{t+1}\right)\right)\right) d \zeta_{t}
$$

As previously discussed, the second marginal density associated with $h_{t}$ is $g_{t}$ and so $\int h_{t}\left(\zeta_{t}, \zeta_{t+1}\right) d \zeta_{t}=$ 
$g_{t}\left(\zeta_{t+1}\right)$. It follows that

$$
p\left(\vartheta_{t+1} \mid X^{t}\right)=\int \mathfrak{h}_{t}\left(\vartheta_{t}, \vartheta_{t+1}\right) d \vartheta_{t}=\mathfrak{g}_{t}\left(\vartheta_{t+1}\right)
$$

and so the conditional distribution of $\vartheta_{t}$, given $X^{t}$, is the TG $\left(A_{t}, B_{t} ; \underline{\vartheta}, \bar{\vartheta}\right)$ where $A_{t}=\omega a_{t}=\omega\left(A_{t-1}+\frac{1}{2}\right)$ and $B_{t}=\omega b_{t}=\omega\left(B_{t-1}+\frac{\left(X_{t}-\mu\right)^{2}}{2}\right)$.

Proof of Theorem 2: By analogy with Weitzman (2007), the predictive distribution of $X_{t+1}$ given $X^{t}$ is given by (later we map into back into the predictive density of $Y_{t+1}=\left(X_{t+1}-\mu\right) / \sqrt{B_{t} A_{t}^{-1}}$ given $\left.X^{t}\right)$,

$$
\begin{aligned}
g\left(X_{t+1} \mid X^{t}\right) & =\int p\left(X_{t+1} \mid \vartheta_{t+1}\right) p\left(\vartheta_{t+1} \mid X^{t}\right) d \vartheta_{t+1} \\
& \propto \int_{\underline{\vartheta}}^{\bar{\vartheta}} \vartheta_{t+1}^{\frac{1}{2}} \exp \left(-\frac{\vartheta_{t+1}\left(X_{t+1}-\mu\right)^{2}}{2}\right) \vartheta_{t+1}^{A_{t}-1} e^{-B_{t} \vartheta_{t+1}} d \vartheta_{t+1} \\
& =\int_{\underline{\vartheta}}^{\vartheta} \vartheta_{t+1}^{\left(A_{t}+\frac{1}{2}\right)-1} \exp \left(-\left(B_{t}+\frac{\left(X_{t+1}-\mu\right)^{2}}{2}\right) \vartheta_{t+1}\right) d \vartheta_{t+1}
\end{aligned}
$$

Since $v_{t}=2 A_{t}$, note that $B_{t}+\frac{\left(X_{t+1}-\mu\right)^{2}}{2}=B_{t}\left(1+\frac{Y_{t+1}^{2}}{v_{t}}\right)$. Using the transformation $u=B_{t}\left(1+\frac{Y_{t+1}^{2}}{v_{t}}\right) \vartheta_{t+1}$, we obtain that

$$
\begin{aligned}
g\left(X_{t+1} \mid X^{t}\right) & \propto \frac{1}{\left(B_{t}\left(1+\frac{Y_{t+1}^{2}}{v_{t}}\right)\right)^{\frac{v_{t}+1}{2}}} \int_{\underline{\vartheta} B_{t}\left(1+Y_{t+1}^{2} / v_{t}\right)}^{\bar{\vartheta} B_{t}\left(1+Y_{t+1}^{2} / v_{t}\right)} u^{\frac{v_{t}+1}{2}-1} e^{-u} d u \\
& \propto \frac{\gamma\left[\frac{v_{t}+1}{2}, 0.5 \overline{\xi_{t}}\left(1+\frac{Y_{t+1}^{2}}{v_{t}}\right)\right]-\gamma\left[\frac{v_{t}+1}{2}, 0.5 \underline{\xi_{t}}\left(1+\frac{Y_{t+1}^{2}}{v_{t}}\right)\right]}{\left(1+\frac{Y_{t+1}^{2}}{v_{t}}\right)^{\frac{v_{t}+1}{2}}},
\end{aligned}
$$

where $\underline{\xi}_{t}=2 \underline{\vartheta} B_{t}, \overline{\xi_{t}}=2 \bar{\vartheta} B_{t}$, and $\gamma[\rho, \kappa]=\int_{0}^{\kappa} z^{\rho-1} e^{-z} d z$ is the lower incomplete Gamma function. Hence, $Y_{t+1}=\left(X_{t+1}-\mu\right) / \sqrt{B_{t} A_{t}^{-1}}$ follows a dampened $t$ distribution with $v_{t}$ degrees of freedom and truncation parameters $\underline{\xi}_{t}$ and $\bar{\xi}_{t}$.

Proof of an Alternative Representation of the Predictive Density of $Y_{t+1}$ in (26):

\section{Lemma 2 Define,}

$$
Y=\frac{Z}{\sqrt{W / v}}
$$


where,

- the random variable $Z$ follows a standard Normal distribution;

- the random variable $W$ follows a $\chi^{2}$ distribution with $\mathrm{v}$ degrees of freedom truncated on the interval $(\underline{\xi}, \bar{\xi})$, where $0<\underline{\xi}<\bar{\xi}<+\infty$;

- $Z$ and $W$ are independent random variables.

Then $Y$ has a density function that coincides with the predictive density (26) obtained from an asset pricing model subject to structural uncertainty.

Proof: We present a proof of this isomorphism as the construction allows us to establish that the moment generating function of $Y_{t+1}$ is well-defined. The densities of $Z$ and $W$ are given by,

$$
\begin{array}{ll}
f_{Z}(z)=\frac{1}{\sqrt{2 \pi}} e^{-\frac{z^{2}}{2}}, & z \in \Re, \\
f_{W}(w)=\frac{1}{c(v, \underline{\xi}, \bar{\xi})} w^{\frac{v}{2}-1} e^{-\frac{w}{2}}, & \underline{\xi}<w<\bar{\xi}
\end{array}
$$

where $c[v, \underline{\xi}, \bar{\xi}]=2^{v / 2}(\gamma[v / 2, \bar{\xi} / 2]-\gamma[v / 2, \underline{\xi} / 2])$ ensures that $f_{W}(w)$ is a proper density. Define the random variable $R=\sqrt{\frac{W}{v}}$. The density of $R$ is seen to be,

$$
f_{R}(r)=2 v r f_{W}\left(v r^{2}\right)=\frac{2 v^{\frac{v}{2}}}{c[\nu, \underline{\xi}, \bar{\xi}]} r^{v-1} e^{-\frac{v r^{2}}{2}}, \quad \sqrt{\underline{\xi} / v}<r<\sqrt{\bar{\xi} / v} .
$$

Adopt the following two transformations,

$$
Y=\frac{Z}{R}, \quad Q=R,
$$

so that $(Y, Q)=g(Z, R)$ where $g(z, r)=(z / r, r)$. A transformation argument using the Jacobian of $g^{-1}$ 
yields that the joint density of $(Y, Q)$ is

$$
\begin{aligned}
f_{Y, Q}(y, q) & =f_{Z, R}(y q, q) q=f_{Z}(y q) f_{R}(q) q, \\
& =\frac{1}{\sqrt{2 \pi}} e^{-\frac{(y q)^{2}}{2}} \frac{2 v^{\frac{v}{2}}}{c[v, \underline{\xi}, \bar{\xi}]} q^{v-1} e^{-\frac{v q^{2}}{2}} q, \\
& =\frac{1}{\sqrt{2 \pi}} \frac{2 v^{\frac{v}{2}}}{c[v, \underline{\xi}, \bar{\xi}]} q^{v} e^{-\frac{\left(v+y^{2}\right) q^{2}}{2}},
\end{aligned}
$$

for $y \in \Re$ and $\sqrt{\underline{\xi} / \nu}<q<\sqrt{\bar{\xi} / \nu}$. To obtain the density of $Y$, integrate $f_{Y, Q}(y, q)$ over $q$ as,

$$
\begin{aligned}
f_{Y}(y) & =\int_{\sqrt{\underline{\xi} / v}}^{\sqrt{\bar{\xi} / v}} f_{Y, Q}(y, q) d q \\
& =\frac{1}{\sqrt{2 \pi}} \frac{2 v^{\frac{v}{2}}}{c[v, \underline{\xi}, \bar{\xi}]} \int_{\sqrt{\underline{\xi} / v}}^{\sqrt{\bar{\xi} / v}} q^{v} e^{-\frac{\left(v+y^{2}\right) q^{2}}{2}} d q .
\end{aligned}
$$

Letting $s=\frac{\left(v+y^{2}\right) q^{2}}{2}$ we obtain $q=\left(\frac{2}{v+y^{2}}\right)^{\frac{1}{2}} s^{\frac{1}{2}}$ and $d q=\frac{1}{2}\left(\frac{2}{v+y^{2}}\right)^{\frac{1}{2}} s^{-\frac{1}{2}} d s$. Therefore,

$$
\begin{aligned}
\int_{\sqrt{\underline{\xi} / v}}^{\sqrt{\bar{\xi} / v}} q^{v} e^{-\frac{\left(v+y^{2}\right) q^{2}}{2}} d q & =\int_{\frac{\xi}{2}\left(1+\frac{y^{2}}{v}\right)}^{\frac{\bar{\xi}}{2}\left(1+\frac{y^{2}}{v}\right)}\left(\frac{2}{v+y^{2}}\right)^{\frac{v}{2}} s^{\frac{v}{2}} e^{-s} \frac{1}{2}\left(\frac{2}{v+y^{2}}\right)^{\frac{1}{2}} s^{-\frac{1}{2}} d s \\
& =\frac{2^{\frac{v-1}{2}}}{\left(v+y^{2}\right)^{\frac{v+1}{2}}} \int_{\frac{\xi}{2}\left(1+\frac{y^{2}}{v}\right)}^{\frac{\xi}{2}\left(1+\frac{y^{2}}{v}\right)} s^{\frac{v+1}{2}-1} e^{-s} d s
\end{aligned}
$$

Accordingly,

$$
\int_{\sqrt{\underline{\xi} / v}}^{\sqrt{\xi / v}} q^{v} e^{-\frac{\left(v+y^{2}\right) q^{2}}{2}} d q=\left(\frac{2^{\frac{v-1}{2}}}{v^{\frac{v+1}{2}}}\right) \frac{\left(\gamma\left[\frac{v+1}{2}, 0.5 \bar{\xi}\left(1+\frac{y^{2}}{v}\right)\right]-\gamma\left[\frac{v+1}{2}, 0.5 \underline{\xi}\left(1+\frac{y^{2}}{v}\right)\right]\right)}{\left(1+\frac{y^{2}}{v}\right)^{\frac{v+1}{2}}}
$$

Substitution of (A21) into (A19) along with the fact that $c[v, \underline{\xi}, \bar{\xi}]=2^{v / 2}(\gamma[v / 2, \bar{\xi} / 2]-\gamma[v / 2, \underline{\xi} / 2])$ proves our assertion. That is,

$$
f_{Y}(y)=\frac{\gamma\left[\frac{v+1}{2}, 0.5 \bar{\xi}\left(1+\frac{Y_{t+1}^{2}}{v}\right)\right]-\gamma\left[\frac{v+1}{2}, 0.5 \underline{\xi}\left(1+\frac{Y_{t+1}^{2}}{v}\right)\right]}{\sqrt{\pi v}(\gamma[v / 2, \bar{\xi} / 2]-\gamma[v / 2, \underline{\xi} / 2])\left(1+\frac{Y_{t+1}^{2}}{v}\right)^{\frac{v+1}{2}}} .
$$


Therefore, the random variable $Y=\frac{Z}{\sqrt{W / v}}$ has the same density as the predictive density of $Y_{t+1}$ derived in (26).

Proof of Theorem 3: Rather than use the predictive density (26) to derive the moment generating function, we appeal to the properties of $Y=\frac{Z}{\sqrt{W / v}}$, which shares the same density function as shown in Lemma 2. Recall $Z$ and $W$ are independent random variables, where $Z$ is distributed standard Normal distribution and $W$ is distributed truncated chi-square distribution. Based on the law of iterated expectations,

$$
\begin{aligned}
E\left(e^{\lambda Y}\right) & =E(\exp (\lambda Z / \sqrt{W / v})) \\
& =E\left(E\left(\exp \left(\left(\frac{\lambda \sqrt{v}}{\sqrt{W}}\right) Z\right) \mid W\right)\right) \\
& =E\left(\exp \left(\frac{\lambda^{2} v}{2 W}\right)\right), \quad \text { (from the mgf of standard normal) }
\end{aligned}
$$

where the expectation in (A25) is to be taken with respect to the chi-squared distribution. Verifying (34) we have,

$$
E\left(e^{\lambda Y}\right)=E\left(\exp \left(\frac{\lambda^{2} v}{2 W}\right)\right)=\frac{1}{c[v, \underline{\xi}, \bar{\xi}]} \int_{\underline{\xi}}^{\bar{\xi}} e^{\frac{\lambda^{2} v}{2 w}} w^{\frac{v}{2}-1} e^{-\frac{w}{2}} d w
$$

where $c[v, \underline{\xi}, \bar{\xi}]=2^{v / 2}(\gamma[v / 2, \bar{\xi} / 2]-\gamma[v / 2, \underline{\xi} / 2])$ is a constant of integration. Now,

$$
\begin{aligned}
E\left(Y^{2 \phi}\right) & =E\left((\lambda Z / \sqrt{W / v})^{2 \phi}\right)=v^{\phi} E\left(Z^{2 \phi}\right) E\left(\frac{1}{W^{\phi}}\right)=v^{\phi} \frac{(2 \phi) !}{\phi ! 2^{\phi}} E\left[\frac{1}{W^{\phi}}\right] \\
& =\left(\frac{v}{2}\right)^{\phi} \frac{(2 \phi) !}{\phi !} \frac{1}{c[v, \underline{\xi}, \bar{\xi}]} \int_{\underline{\xi}}^{\bar{\xi}} w^{-\phi} w^{\frac{v}{2}-1} e^{-\frac{w}{2}} d w .
\end{aligned}
$$

Finally, the odd-order moments of $Y$ are zero since the density is symmetric around zero.

Proof of the Rare Event Probabilities in Equation (40): Let $x^{\text {rare }}=\mu-h \sqrt{V}$, where the volatility 
multiple $h$ represents the deviation from mean consumption growth. Thus, from Lemma 2,

$$
\begin{aligned}
\operatorname{Prob}\left(X_{t+1} \leq x^{\mathrm{rare}}\right) & =\operatorname{Prob}\left(Y_{t+1} \leq-h\right)=\operatorname{Prob}(Z \sqrt{v / W} \leq-h) \\
& =E(\operatorname{Prob}(Z \leq-h \sqrt{W / v} \mid W)) \\
& =E(N(-h \sqrt{W / v})), \\
& =\frac{1}{c[v, \underline{\xi}, \bar{\xi}]} \int_{\underline{\xi}}^{\bar{\xi}} N(-h \sqrt{w / v}) w^{\frac{v}{2}-1} e^{-\frac{w}{2}} d w
\end{aligned}
$$

where $N(d)=\frac{1}{\sqrt{2 \pi}} \int_{-\infty}^{d} e^{-u^{2} / 2} d u$ is the Normal probability integral. 


\section{References}

Abel, Andrew, 2002, An exploration of the effects of pessimism and doubt on asset returns, Journal of Economic Dynamics and Control 26, 1075-1092.

Adam, Klaus, Albert Marcet, and Juan Nicolini, 2008, Stock market volatility and learning, Working paper, European Central Bank.

Aït-Sahalia, Yacine, Jonathan Parker, and Motohiro Yogo, 2004, Luxury goods and the equity premium, Journal of Finance 59(6), 2959-3004.

Bansal, Ravi, and Amir Yaron, 2004, Risks for the long run: A potential resolution of asset pricing puzzles, Journal of Finance 59, 1481-1509.

Barro, Robert, 2006, Rare disasters and asset markets in the twentieth century, Quarterly Journal of Economics $121,823-866$.

Barsky, Robert, and Bradford DeLong, 1993, Why does the stock market fluctuate?, Quarterly Journal of Economics 108, 291-311.

Bauwens, Luc, Michel Lubrano, and Jean-Francois Richard, 1999, Bayesian Inference in Dynamic Econometric Models. (Oxford University Press New York, NY.).

Brennan, Michael, and Yihong Xia, 2001, Stock price volatility and equity premium, Journal of Monetary Economics 47, 249-283.

Campbell, John, 1986, Bond and stock returns in a simple exchange model, Quarterly Journal of Economics $101,785-804$.

Campbell, John, 1993, Intertemporal asset pricing without consumption data, American Economic Review $83,487-512$.

Campbell, John, and John Cochrane, 1999, Force of habit: A consumption-based explanation of aggregate stock market behavior, Journal of Political Economy 107, 205-251.

Campbell, John, and John Cochrane, 2000, Explaining the poor performance of consumption-based asset pricing models, The Journal of Finance 55, 2683-2878. 
Cecchetti, Stephen, Pok-Sang Lam, and Nelson Mark, 2000, Asset pricing with distorted beliefs: Are equity returns too good to be true?, American Economic Review 90, 787-805.

Coffey, Christopher, and Keith Muller, 2000, Properties of doubly-truncated Gamma variables, Communications in Statistics - Theory and Methods 29, 851-857.

Cogley, Timothy, 2007, Is the market price of risk infinite?, Working paper, Department of Economics, University of California-Davis.

Cogley, Timothy, and Thomas Sargent, 2008, The market price of risk and the equity premium: A legacy of the great depression?, Working paper, Department of Economics, University of California-Davis and New York University.

Constantinides, George, 1990, Habit formation: A resolution of the equity premium puzzle, Journal of Political Economy 98, 519-543.

Cox, John, Jonathan Ingersoll, and Stephen Ross, 1985, An intertemporal general equilibrium model of asset prices, Econometrica 53, 363-384.

Duffie, Darrell, 1992, Dynamic Asset Pricing Theory. (Princeton University Press Princeton, New Jersey) 2nd edn.

Epstein, L., and S. Zin, 1991, Substitution, risk aversion, and the temporal behavior of consumption and asset returns: An empirical analysis, Journal of Political Economy 99, 263-286.

Gabaix, Xavier, 2007, Variable rare disasters: An exactly solved framework for ten puzzles in macrofinance, Working paper, Stern School of Business, New York University.

Geweke, John, 2001, A note on some limitations of CRRA utility, Economics Letters 71, 341-345.

Hansen, Lars, and Ravi Jagannathan, 1991, Implications of security market data for dynamic economies, Journal of Political Economy 99, 225-261.

Harrison, Michael, and David Kreps, 1979, Martingales and arbitrage in multiperiod securities markets, Journal of Economic Theory 20, 381-408.

Harvey, Andrew, and C. Fernandes, 1989, Time series model for insurance claims, Journal of the Institute of Actuaries 116, 513-528. 
Jobert, A., A. Platania, and L. C. G. Rogers, 2006, A Bayesian solution to the equity premium puzzle, Working paper, Statistical Laboratory, University of Cambridge.

Kendall, Maurice, and Alan Stuart, 1977, The Advanced Theory of Statistics, Volume 1. (MacMillan Publishing New York).

Kocherlakota, Narayana, 1996, The equity premium: It's still a puzzle, Journal of Economic Literature 34, $42-71$.

LeRoy, Stephen, and Richard Porter, 1981, The present-value relation: Tests based on implied variance bounds, Econometrica 101, 555-574.

Lettau, Martin, Sydney Ludvigson, and Jessica Wachter, 2007, The declining equity premium: What role does macroeconomic risk play?, Review of Financial Studies 21, 1653-1687.

Lettau, Martin, and Jessica Wachter, 2007, Why is long-horizon equity less risky? A duration-based explanation of the value premium, Journal of Finance 62, 55-92.

Lewellen, Jonathan, and Jay Shanken, 2002, Learning, asset-pricing tests, and market efficiency, Journal of Finance 57 (2), 1113-1145.

Lucas, Robert, 1978, Asset prices in an exchange economy, Econometrica 46, 1429-1445.

Lukacs, Eugene, 1960, Characteristic Functions. (Charles Griffin and Company London).

Mehra, Rajnish, and Edward Prescott, 1985, The equity premium: A puzzle, Journal of Monetary Economics 15(2), 145-161.

Mehra, Rajnish, and Edward Prescott, 2003, The equity premium in retrospect, in George Constantinides, Milton Harris, and Rene Stulz, eds.: Handbook of the Economics of Finance (Elsevier Science, North Holland: Amsterdam ).

Mehra, Rajnish, and Edward Prescott, 2008, Equity premium, in Rajnish Mehra, eds.: The Handbook of the Equity Risk Premium (Elsevier Science, North Holland: Amsterdam ).

Merton, Robert, 1969, Lifetime portfolio selection under uncertainty: The continuous time case, Review of Economics and Statistics 51, 247-257. 
Rietz, Thomas, 1988, The equity risk premium: A solution, Journal of Monetary Economics 22, 117-131.

Shephard, Neil, 1994, Local scale models: State space alternative to integrated GARCH processes, Journal of Econometrics 60, 181-202.

Timmermann, Allan, 1993, How learning in financial markets generate excess volatility and predictability in stock prices, Quarterly Journal of Economics 108, 1135-1145.

Timmermann, Allan, 1996, Excess volatility and predictability of stock prices in autoregressive dividend models with learning, Review of Economic Studies 63, 523-557.

Tsionas, Efthymios, 2005, Likelihood evidence on the asset return puzzle, Review of Economic Studies 72, 917-946.

Weil, Philippe, 1989, The equity premium puzzle and the riskfree rate puzzle, Journal of Monetary Economics 24, 401-421.

Weitzman, Martin, 2007, Subjective expectations and asset pricing puzzles, American Economic Review 97, 1102-1130. 
Table 1

Riskfree Return and Equity Premium When the Maximum Consumption Growth Volatility, $\bar{\sigma}$, is $50 \%$

\begin{tabular}{llrrr}
\hline$\alpha$ & $\begin{array}{l}\text { Effective } \\
\text { Sample Size }\end{array}$ & $\begin{array}{r}\text { Riskfree } \\
\text { Return }\end{array}$ & $\begin{array}{r}\text { Expected } \\
\text { Return }\end{array}$ & $\begin{array}{r}\text { Equity } \\
\text { Premium }\end{array}$ \\
\hline \multirow{3}{*}{2} & $k=10$ & $6.14 \%$ & $6.47 \%$ & $0.33 \%$ \\
& $k=30$ & $6.21 \%$ & $6.47 \%$ & $0.26 \%$ \\
& $k=50$ & $6.39 \%$ & $6.47 \%$ & $0.08 \%$ \\
& Normal & $6.39 \%$ & $6.47 \%$ & $0.08 \%$ \\
& & & & \\
3 & $k=10$ & $7.73 \%$ & $8.22 \%$ & $0.49 \%$ \\
& $k=30$ & $7.88 \%$ & $8.27 \%$ & $0.40 \%$ \\
& $k=50$ & $8.28 \%$ & $8.41 \%$ & $0.13 \%$ \\
& Normal & $8.29 \%$ & $8.41 \%$ & $0.12 \%$ \\
5 & & & & \\
& $k=10$ & $10.42 \%$ & $11.24 \%$ & $0.82 \%$ \\
& $k=30$ & $10.82 \%$ & $11.48 \%$ & $0.66 \%$ \\
& $k=50$ & $11.90 \%$ & $12.20 \%$ & $0.21 \%$ \\
& Normal & $11.97 \%$ & $12.17 \%$ & $0.20 \%$ \\
10 & & & & \\
& $k=10$ & $14.12 \%$ & $15.82 \%$ & $1.70 \%$ \\
& $k=30$ & $15.85 \%$ & $17.18 \%$ & $1.33 \%$ \\
& $k=50$ & $20.40 \%$ & $20.80 \%$ & $0.42 \%$ \\
& Normal & $20.47 \%$ & $20.87 \%$ & $0.40 \%$ \\
& & & & \\
\hline
\end{tabular}

Reported are the riskfree return and the equity premium under structural uncertainty and Bayesian learning. The computation rely on the following parameter inputs:

$$
\mu=2 \%, \quad \sqrt{V}=2 \%, \quad k \in\{10,30,50\}, \quad \beta=0.99, \quad \underline{\sigma}=1 / \sqrt{\bar{\vartheta}}=0.1 \%, \quad \bar{\sigma}=1 / \sqrt{\vartheta}=50 \% .
$$

The Normal distribution corresponds to the case of no structural uncertainty and reported in the row "Normal." We compute the riskfree return as

$$
\begin{aligned}
\ln \left(R_{t+1}^{f}\right) & =-\ln \beta-\ln \Psi_{X}[-\alpha] \\
& =-\ln \beta+\mu \alpha-\ln \left(\frac{1}{c[v, \underline{\xi}, \bar{\xi}]} \int_{\underline{\xi}}^{\bar{\xi}} e^{\frac{\alpha^{2} V v}{2 w}} w^{\frac{v}{2}-1} e^{-\frac{w}{2}} d w\right)
\end{aligned}
$$

and the equity risk premium as

$$
\ln \left(E_{t} R_{t+1}^{e}\right)-\ln \left(R_{t+1}^{f}\right)=\ln \Psi_{X}[1]-\ln \Psi_{X}[1-\alpha]+\ln \Psi_{X}[-\alpha]
$$

where $\Psi_{X}[\lambda]$ is presented in equation (35) of Theorem 3 . The equity premium and the riskfree return are identical when $\bar{\sigma}=100 \%$ and not reported. 
Table 2

Matching the Riskfree Return and the Equity Premium Using the Asset Pricing Model with Subjective Expectations

\begin{tabular}{|c|c|c|c|c|c|}
\hline \multicolumn{3}{|c|}{$\begin{array}{c}\text { Panel A: Matched } \\
\text { Equity Premium of } 6 \%\end{array}$} & \multicolumn{3}{|c|}{$\begin{array}{l}\text { Panel B: Matched Riskfree } \\
\text { Return of } 1 \%\end{array}$} \\
\hline$\alpha$ & $\bar{\sigma}$ & $\begin{array}{r}\text { Riskfree } \\
\text { Return }\end{array}$ & $\alpha$ & $\bar{\sigma}$ & $\begin{array}{r}\text { Equity } \\
\text { Premium }\end{array}$ \\
\hline 2 & 11.935 & $0.47 \%$ & 2 & 11.932 & $5.47 \%$ \\
\hline 3 & 7.651 & $2.41 \%$ & 3 & 7.655 & $7.41 \%$ \\
\hline 4 & 5.569 & $4.30 \%$ & 4 & 5.575 & $9.30 \%$ \\
\hline 5 & 4.346 & $6.16 \%$ & 5 & 4.353 & $11.16 \%$ \\
\hline 6 & 3.546 & $7.97 \%$ & 6 & 3.553 & $12.97 \%$ \\
\hline 7 & 2.983 & $9.74 \%$ & 7 & 2.990 & $14.74 \%$ \\
\hline 8 & 2.566 & $11.47 \%$ & 8 & 2.573 & $16.47 \%$ \\
\hline 9 & 2.246 & $13.15 \%$ & 9 & 2.253 & $18.15 \%$ \\
\hline 10 & 1.992 & $14.80 \%$ & 10 & 2.000 & $19.80 \%$ \\
\hline 15 & 1.251 & $22.40 \%$ & 15 & 1.258 & $27.40 \%$ \\
\hline 20 & 0.893 & $28.94 \%$ & 20 & 0.900 & $33.94 \%$ \\
\hline
\end{tabular}

Reported are the return on the riskfree bond and the equity premium under structural uncertainty and Bayesian learning by varying the maximum level of consumption growth volatility $\bar{\sigma}=1 / \sqrt{\underline{\vartheta}}$ and the risk aversion $\alpha$ along possible grid points. Fixing $\alpha, \bar{\sigma}=1 / \sqrt{\underline{\vartheta}}$ is varied to match the equity premium of $6 \%$ in Panel A, and the riskfree return of $1 \%$ in Panel B. The computation rely on the following parameters:

$$
\mu=2 \%, \quad \sqrt{V}=2 \%, \quad k=50, \quad \beta=0.99, \quad \underline{\sigma}=1 / \sqrt{\bar{\vartheta}}=0.1 \% .
$$

The equity premium and the riskfree return are both expressed as annual percentages. Note that the maximum allowable consumption growth volatility $\bar{\sigma}$ is not expressed in percentage terms (i.e., $\bar{\sigma}=11.935$ connotes $1193.5 \%)$. 
Table 3

Structural Uncertainty and the Probability of Rare Disasters

\begin{tabular}{cccccc}
\hline$h$ & $\begin{array}{c}\text { Normal } \\
\text { Distribution }\end{array}$ & $\bar{\sigma}=4 \%$ & $\bar{\sigma}=5 \%$ & $\bar{\sigma}=6 \%$ & $\begin{array}{c}\text { Dampened t Distribution } \\
\text { Distribution }\end{array}$ \\
\hline 3 & $1.349898 \mathrm{E}-03$ & $2.100851 \mathrm{E}-03$ & $2.100852 \mathrm{E}-03$ & $2.100852 \mathrm{E}-03$ & $2.100852 \mathrm{E}-03$ \\
4 & $3.167124 \mathrm{E}-05$ & $1.045948 \mathrm{E}-04$ & $1.045951 \mathrm{E}-04$ & $1.045951 \mathrm{E}-04$ & $1.045951 \mathrm{E}-04$ \\
5 & $2.866516 \mathrm{E}-07$ & $3.716510 \mathrm{E}-06$ & $3.716606 \mathrm{E}-06$ & $3.716606 \mathrm{E}-06$ & $3.716606 \mathrm{E}-06$ \\
6 & $9.865877 \mathrm{E}-10$ & $1.094241 \mathrm{E}-07$ & $1.094470 \mathrm{E}-07$ & $1.094470 \mathrm{E}-07$ & $1.094470 \mathrm{E}-07$ \\
\hline
\end{tabular}

The table presents the probability of rare disasters corresponding to the (i) Normal distribution, (ii) the dampened t distribution by varying the maximum level of consumption growth volatility $\bar{\sigma}$, and (iii) the Student-t distribution. Rare disaster $x^{\text {rare }}$ represents the deviation from mean consumption growth in multiples of volatility:

$$
x^{\mathrm{rare}}=\mu-h \sqrt{V},
$$

where $h \in\{3,4,5,6\}, \mu=2 \%$, and $\sqrt{V}=2 \%$. Under the assumption that $k=50$ and $\underline{\sigma}=1 / \sqrt{\vartheta}=0.1 \%$, we compute and report the rare disaster probabilities:

$$
\operatorname{Prob}\left(X_{t+1} \leq x^{\mathrm{rare}}\right) \frac{1}{c[v, \underline{\xi}, \bar{\xi}]} \int_{\underline{\xi}}^{\bar{\xi}} N(-h \sqrt{w / v}) w^{\frac{v}{2}-1} e^{-\frac{w}{2}} d w
$$

where $v=k-1$ is the degrees of freedom, $\underline{\xi}=\frac{(k-1) V}{\bar{\sigma}^{2}}, \bar{\xi}=\frac{(k-1) V}{\underline{\sigma}^{2}}$, and $N(d)=\frac{1}{\sqrt{2 \pi}} \int_{-\infty}^{d} e^{-u^{2} / 2} d u$ is the Normal probability integral. The Normal distribution probabilities correspond to $k=\infty$, and the Student-t distribution probabilities correspond to $\underline{\sigma}=1 / \sqrt{\bar{\vartheta}}=0$ and $\bar{\sigma}=1 / \sqrt{\vartheta}=\infty$. 

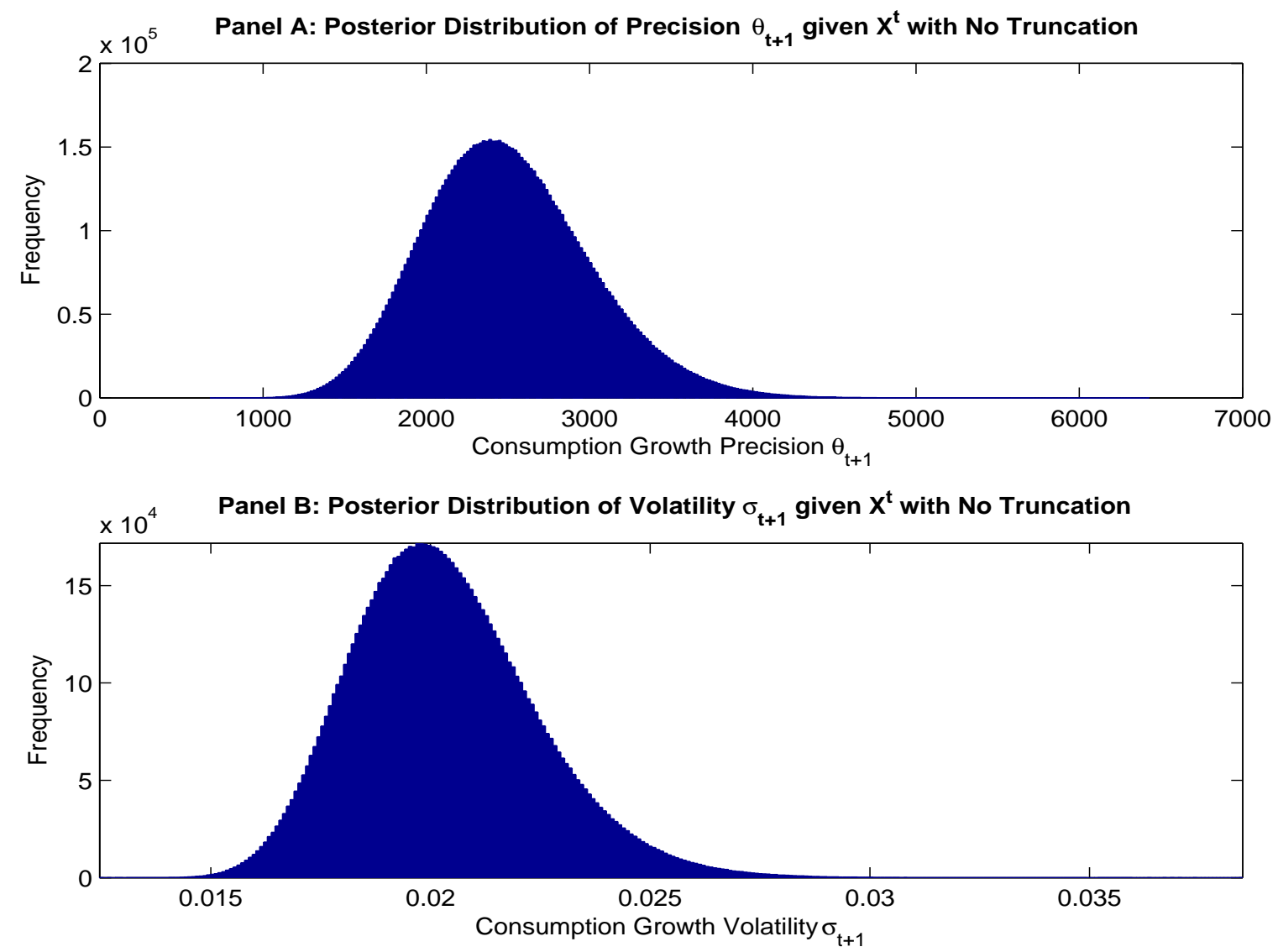

Fig. 1. Simulating the Posterior Distribution of $\theta_{t+1}$ and $\sigma_{t+1}$ given $X^{t}$

Plotted here is the posterior distribution of $\theta_{t+1}$ given $X^{t}$ under no truncation, which is $\operatorname{Gamma}(A, B)$ given the availability of a large past history. In the computations, the volatility of consumption growth is $\sqrt{V}=2 \%$ and the effective sample size is $k=50$. Therefore,

$$
A=(k-1) / 2=24.5, \quad B=(k-1) V / 2=0.0098 .
$$

Panel A presents the distribution of the precision of consumption growth, $\theta_{t+1}$, while Panel B presents the distribution of the volatility of consumption growth, $\sigma_{t+1}=1 / \sqrt{\theta_{t+1}}$. Both plots are based on a simulation of 10 million draws. 


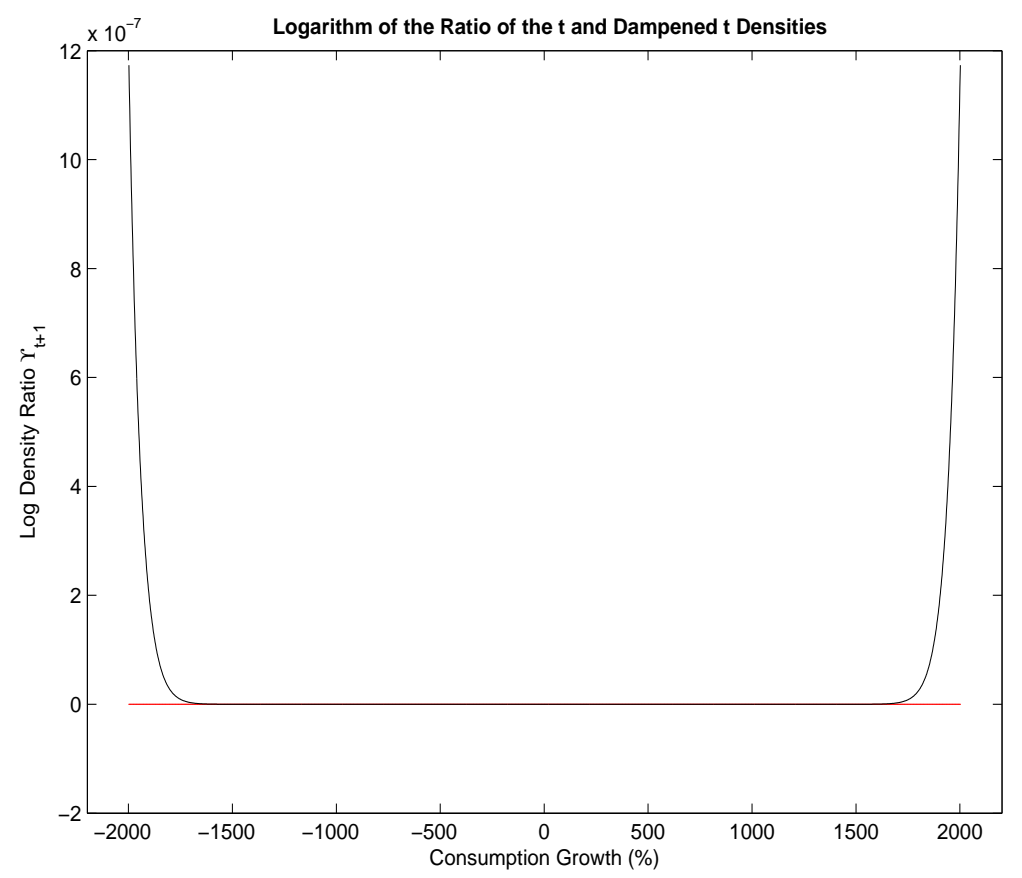

Fig. 2. Plot of $\ln \left(\frac{g\left(X_{t+1} ; k-1\right)}{g^{\mathrm{DT}}\left(X_{t+1} ; k-1, \underline{\vartheta}(k-1) V, \bar{\vartheta}(k-1) V\right)}\right)$ versus $X_{t+1}$

Plotted is the logarithm ratio of densities defined below (under the availability of large past history):

$$
\Upsilon_{t+1} \equiv \ln \left(\frac{g\left(X_{t+1} ; k-1\right)}{g^{\mathrm{DT}}\left(X_{t+1} ; k-1, \underline{\vartheta}(k-1) V_{t}, \bar{\vartheta}(k-1) V_{t}\right)}\right), \quad X_{t+1}=\mu+\sqrt{V_{t}} Y_{t+1},
$$

as a function of consumption growth $X_{t+1}$. Given that $Y_{t+1}$ is Student t-distributed based on (14)-(15), the density of $X_{t+1}$ is seen to be:

$$
g\left(X_{t+1} ; k-1\right)=\frac{1}{\sqrt{V}} \frac{\Gamma[k / 2]}{\sqrt{\pi(k-1)} \Gamma[(k-1) / 2]}\left(1+\frac{\left(\left(X_{t+1}-\mu\right) / \sqrt{V}\right)^{2}}{k-1}\right)^{-k / 2} .
$$

The form of density $g^{\mathrm{DT}}\left(X_{t+1} ; k-1, \underline{\vartheta}(k-1) V, \bar{\vartheta}(k-1) V\right)$ is similarly obtained, as $Y_{t+1}$ follows the dampened $t$ distribution with density presented in (26). Here $v=2 A=k-1, \underline{\xi}=\underline{\vartheta}(k-1) V$ and $\bar{\xi}=\bar{\vartheta}(k-1) V$. For the purposes of this graph,

$$
\mu=2 \%, \quad \sqrt{V}=2 \%, \quad k=50, \quad \underline{\sigma}=1 / \sqrt{\bar{\vartheta}}=0.01 \%, \quad \bar{\sigma}=1 / \sqrt{\underline{\vartheta}}=500 \% \text {. }
$$



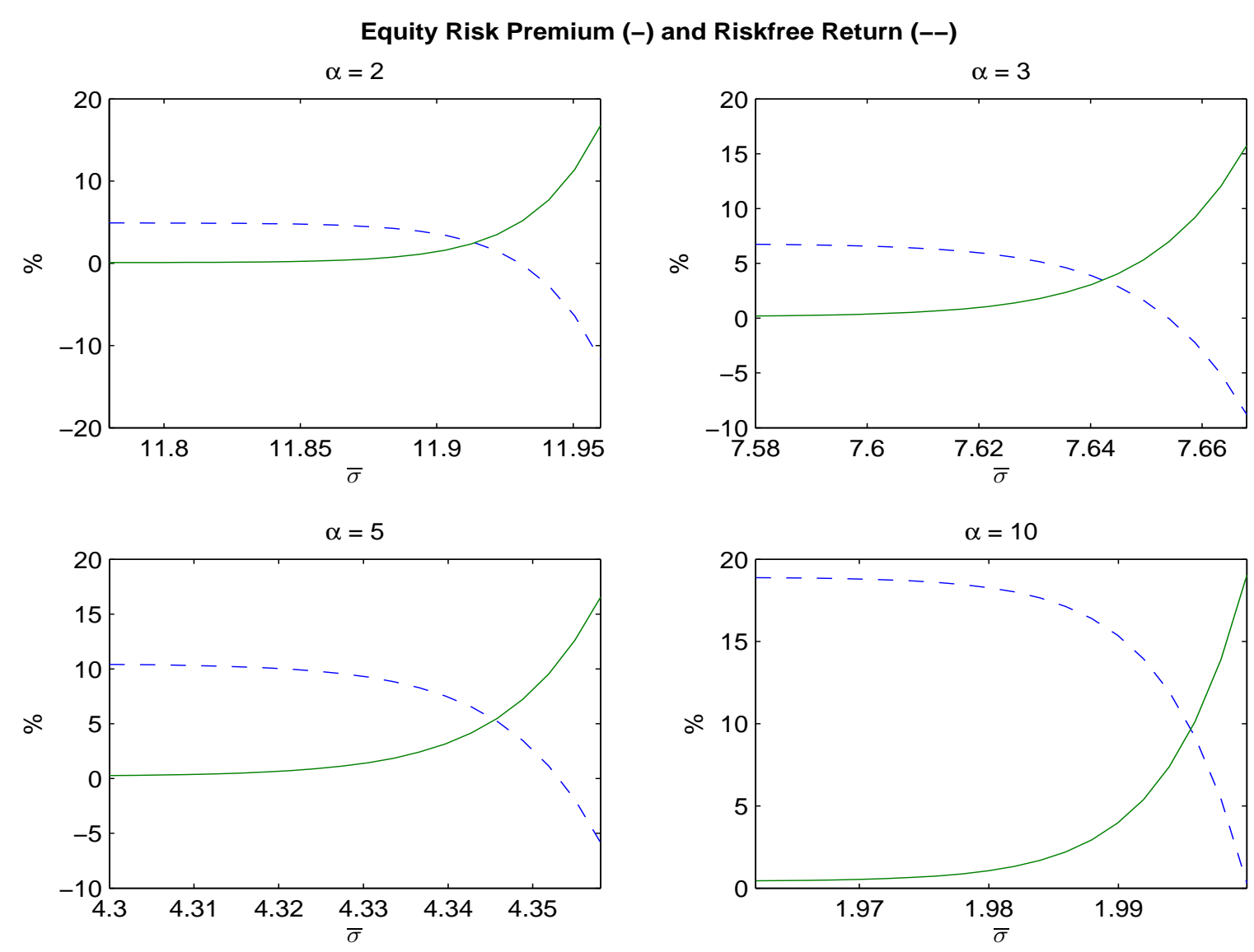

Fig. 3. Equity Premium and Riskfree Return versus Maximum Consumption Growth Volatility $\bar{\sigma}$

The dashed-curve depicts the riskfree return, and the solid-curve depicts the equity risk premium on the $\mathrm{y}$-axis, while changing $\bar{\sigma}$ on the $\mathrm{x}$-axis. Note that the value, say, 11.9 on the $\mathrm{x}$-axis corresponds to $1,190 \%$. For these plots we set,

$$
\mu=2 \%, \quad \sqrt{V}=2 \%, \quad k=50, \quad \beta=0.99, \quad \underline{\sigma}=1 / \sqrt{\bar{\vartheta}}=0.01 \% .
$$

Each plot corresponds to fixed risk aversion $\alpha \in\{2,3,5,10\}$. We compute the riskfree return as

$$
\begin{aligned}
\ln \left(R_{t+1}^{f}\right) & =-\ln \beta-\ln \Psi_{X}[-\alpha] \\
& =-\ln \beta+\mu \alpha-\ln \left(\frac{1}{c[v, \underline{\xi}, \bar{\xi}]} \int_{\underline{\xi}}^{\bar{\xi}} e^{\frac{\alpha^{2} V v}{2 w}} w^{\frac{v}{2}-1} e^{-\frac{w}{2}} d w\right)
\end{aligned}
$$

and the equity premium as

$$
\ln \left(E_{t} R_{t+1}^{e}\right)-\ln \left(R_{t+1}^{f}\right)=\ln \Psi_{X}[1]-\ln \Psi_{X}[1-\alpha]+\ln \Psi_{X}[-\alpha]
$$

where $\Psi_{X}[\lambda]$ is presented in equation (35) of Theorem 3 . 